Pacific Journal of Mathematic 


\section{SEQUENTIAL TESTING OF SEVERAL SIMPLE HYPOTHESES FOR A DIFFUSION PROCESS AND THE CORRESPONDING FREE BOUNDARY PROBLEM}

\section{Luis A. Caffarelli and Avner Friedman}

An $n$-dimensional stochastic process $\xi(t)$ is observed. It is known that $\xi(t)$ has the statistics of an $n$-dimensional Brownian motion with any one of possibly $n+1$ drifts $\lambda_{0}, \cdots, \lambda_{n}$ ( $\lambda_{i}$ are given $n$-vectors). We observe the process at a running cost, per unit time, given by $c_{i}$ when the drift is $\lambda_{i}$, and after some (stopping) time $\tau$ make a decision which hypothesis to accept; the hypothesis $H_{j}$ means accepting the drift $\lambda_{j}$; the drift changes in time in accordance with a Markov process with $n+1$ states and a given transition probability matrix. The problem of finding the optimal stopping time and optimal final decision leads to a variational inequality for a degenerate elliptic operator. In this paper we study this variational inequality and the corresponding free boundary. We also consider, by purely probabilistic methods, the case where $\xi(t)$ is $k$-dimensional, $k \neq n$. The outline of the main results is given at the end of $\S 2$.

1. The sequential testing problem. Let $q_{i, j}(0 \leqq i, j \leqq n)$ be real numbers such that $q_{i, j} \geqq 0$ if $i \neq j, q_{i, i} \leqq 0$, and $\sum_{j=0}^{n} q_{i, j}=0$ for $0 \leqq i \leqq n$. In a probability space $(\Omega, \mathscr{F}, P)$ we are given a Markov process $\theta(t)=\theta(t, w)$ taking values $0,1, \cdots, n$ and having the infinitesimal matrix $\left(q_{i, j}\right)$. We are also given an $n$-dimensional Brownian motion $w(t)$ (with $w(0)=0$ ) independent of the process $\theta(t)$. Let $\lambda_{0}, \lambda_{1}, \cdots, \lambda_{n}$ be $n$-dimensional vectors which span $R^{n}$, that is

$$
\lambda_{1}-\lambda_{0}, \lambda_{2}-\lambda_{0}, \cdots, \lambda_{n}-\lambda_{0} \text { are linearly independent. }
$$

Consider the process $\xi(t)$ in $R^{n}$ given by

$$
d \xi(t)=d w(t)+\sum_{j=0}^{n} I_{(\theta(t)=j)} \lambda_{j} d t
$$

that is, on the set $\theta(t, \omega)=j d \xi(t, \omega)=d w(t, \omega)+\lambda_{j} d t$. We set $\mathscr{F}_{t}=\sigma(\xi(s), 0 \leqq s \leqq t)$.

When $\theta(t, \omega)=j$ we say that the hypothesis $H_{j}$ is satisfied (at time $t$ ). We shall be concerned with the problem of deciding which hypothesis to accept at a minimal cost. We follow Bayes' formulation in setting up the problem: 
The observed process is $\xi(t)$. We are given an a priori probability $\pi$ for $\theta(0)$, that is, we are given

$$
\pi=\left(\pi_{0}, \pi_{1}, \cdots, \pi_{n}\right), \quad \pi_{i} \geqq 0, \quad \sum_{i=0}^{n} \pi_{i}=1
$$

and make the initial assumption that $\theta(0)=j$ with probability $\pi_{j}$. This determines a probability $P^{\pi}$ on the space of paths $(\theta(t), w(t))$ with $w(0)=0$, and

$$
P^{\pi}(\theta(0)=j)=\pi_{j}, \quad 0 \leqq j \leqq n .
$$

We shall denote the expectation with respect to $P^{\pi}$ by $E^{\pi}$. The running cost (per unit time) of the observation of $\xi(t)$ is a given positive number $c_{j}$ if $\theta(t)=j$. We observe the process $\xi(t)$ for an amount of time $\tau$, where $\tau$ is a stopping time with respect to $\mathscr{F}_{t}$; the incurred cost is then

$$
E^{\pi}\left[\int_{0}^{\tau} f(\theta(t)) d t\right], \quad \text { where } f(j)=c_{j} \quad(0 \leqq j \leqq n) .
$$

At the time $t=\tau$ we make a terminal decision $d(\omega)$ as to which hypothesis to accept; $d(\omega)=j$ means accepting the hypothesis $H_{j}$. The variable $d(\omega)$ is taken to be $\mathscr{F}_{\tau}$ measurable.

Set

$$
W(\theta, d)=a_{i} \quad \text { if } \quad d=i, \theta \neq i \quad\left(a_{i}>0\right),
$$

i.e., $a_{i}$ is the cost for erroneously accepting the hypothesis $H_{i}$. The cost of the terminal decision is

$$
E^{\tau}[W(\theta(\tau, \omega), d(\omega))]
$$

and the total cost for the decision $\delta=(\tau, d)$ is

$$
J_{\pi}(\delta) \equiv E^{\pi}\left[\int_{0}^{\tau} f(\theta(t)) d t+W(\theta(\tau, \omega), d(\omega))\right] .
$$

More generally, introducing a discount factor $\alpha, \alpha \geqq 0$, the total cost becomes

$$
J_{\pi}(\delta)-E^{\pi}\left[\int_{0}^{\tau} e^{-\alpha t} f(\theta(t)) d t+e^{-\alpha \tau} W(\theta(\tau, \omega), d(\omega))\right] .
$$

The problem is to study the least cost function

$$
V(\pi)=\inf _{\delta} J_{\pi}(\delta)
$$

and to find an optimal decision $\tilde{\delta}=(\widetilde{\tau}, \widetilde{d})$, that is,

$$
J_{\pi}(\tilde{\delta})=V(\pi) \text {. }
$$


This problem is called a sequential testing problem of $n+1$ simple hypotheses $H_{0}, H_{1}, \cdots, H_{n}$. The case where

$$
\begin{aligned}
& \theta \text { does not depend on } t \text {, that is, } q_{i, j}=0 \text { for } \\
& 0 \leqq i, j \leqq n ; c_{i}=c>0 \text { for } \leqq i \leqq n
\end{aligned}
$$

will be called the special case; more refined results will be proved for this case.

The sequential testing problem in the special case with $n=2$ has been studied in detail (see Shiryayev [15] and the references given there). In the case of discrete times the problem (in the special case) was studied by Wald [16], Chow and Robins [7], Shiryayev [14], Kiefer and Sacks [11] and others.

Analogously to the case $n=2$ we introduce the a posteriori probability process

$$
\pi(t)=\left(\pi_{0}(t), \pi_{1}(t), \cdots, \pi_{n}(t)\right)
$$

where

$$
\pi_{j}(t)=P^{\pi}\left[\theta(t)=j \mid \mathscr{F}_{t}\right] .
$$

Introducting the simplex in $R^{n+1}$

$$
\Pi_{n+1}=\left\{\pi=\left(\pi_{0}, \pi_{1}, \cdots, \pi_{n}\right) ; \pi_{i} \geqq 0, \sum_{i=1}^{n} \pi_{i}=1\right\}
$$

it is clear that $\pi(t) \in \Pi_{n+1}$ for all $t>0$. The process $\pi(t)$ was studied by Shiryayev (see [13]) and by Anderson and Friedman [2]. It is shown in these references that $\pi(t)$ is a Markov process with generator

$$
\begin{aligned}
& \operatorname{Mu}(\pi)=\frac{1}{2} \sum_{i, j=0}^{n} \pi_{i} \pi_{j}\left(\lambda_{i}-\sum_{k=0}^{n} \lambda_{k} \pi_{k}\right) . \\
& \left(\lambda_{j}-\sum_{l=0}^{n} \lambda_{l} \pi_{l}\right) \frac{\partial^{2} u(\pi)}{\partial \pi_{i} \partial \pi_{j}}+\sum_{i, j=0}^{n} q_{i, j} \pi_{i} \frac{\partial u(\pi)}{\partial \pi_{j}}
\end{aligned}
$$

and (in [2]) explicit formulas are given for $\pi_{j}(t)$ in terms of $\xi(t)$. In particular, when (1.9) holds,

$$
\begin{gathered}
\pi_{j}(t)=\pi_{j}\left\{\sum_{k=0}^{n} \pi_{k} z_{j, k}(t)\right\}^{-1}, \\
z_{j, k}(t)=\exp \left\{\left(\lambda_{k}-\lambda_{j}\right) \cdot \xi(t)-\frac{1}{2}\left(\left.\left\|\left.\lambda_{k}\right|^{2}-\right\| \lambda_{j}\right|^{2}\right) t\right\} .
\end{gathered}
$$

As in [2] [13; p. 167] we can express $J_{\pi}(\delta)$ in terms of the process $\pi(t)$ :

$$
J_{\pi}(\delta)=E^{\pi}\left\{\int_{0}^{\tau} e^{-\alpha t} h(\pi(t)) d t+e^{-\alpha \tau} \sum_{i=0}^{n}\left(1-\pi_{i}(\tau)\right) a_{i} I_{[d(\omega)=i]\}},\right.
$$


where $h(\pi)=\sum_{i=0}^{n} c_{i} \pi_{i}$.

Set

$$
J_{\pi}(\tau)=\inf _{d} J_{\pi}(\delta) \quad \text { where }(\tau, d)=\delta
$$

For a given $\tau$ the optimal $d=d(\omega)$ is such that it minimizes $\sum\left(1-\pi_{i}(\tau(\omega))\right) a_{i}$. Consequently,

$$
J_{\pi}(\tau)=E^{\pi}\left[\int_{0}^{\tau} e^{-\alpha t} h(\pi(t)) d t+e^{-\alpha \tau} g(\pi(\tau))\right]
$$

where

$$
g(\pi)=\min _{0 \leqq i \leqq n}\left\{a_{i}\left(1-\pi_{i}\right)\right\} .
$$

The problem associated with (1.7), (1.8) thus reduces to the problem associated with

$$
V(\pi)=\inf _{\tau} J_{\pi}(\tau)
$$

(where $V(\pi)$ is the same as in (1.17)) and

$$
J_{\pi}(\widetilde{\tau})=V(\pi),
$$

where $\tau, \widetilde{\tau}$ are stopping times with respect to $\mathscr{F}_{t}$.

In the sequel we shall study the hypothesis testing problem in its formulation (1.15), (1.16). For simplicity we shall also always assume that $\alpha>0$; the results in case $\alpha=0$ are still valid, but require some changes in the proofs; we consider this case briefly in $\S 10$.

2. The variational inequality, Let $\check{\Pi}_{n+1}=\operatorname{int} \Pi_{n+1}$.

As in [2], the function $V(\pi)$ in $\Pi_{n+1}$ can be characterized as the bounded solution $u$ of a certain system of differential in equalities:

$$
\begin{gathered}
M u-\alpha u+h \geqq 0 \text { a.e. in } \Pi_{n+1}, \\
u(\pi) \leqq h(\pi) \text { in } \Pi_{n+1}, \\
(M u-\alpha u+h)(u-g)=0 \text { a.e. in } \Pi_{n+1} .
\end{gathered}
$$

Such a system is called a variational inequality (for a general study of variational inequalities see, for instance, [3] [9]).

We recall [2] that, because of (1.1), $M$ is a nondegenerate elliptic operator (in $n$ independent variables) in $\check{\Pi}_{n+1}$. It degenerates however on the boundary $\partial \Pi_{n+1}$.

LEMma 2.1. (a) If $\pi=\pi(0)$ belongs to $\stackrel{\circ}{\Pi}_{n+1}$ then $\pi(t) \in \stackrel{\circ}{\Pi}_{n+1}$ for 
all $t>0$, and (b) if (1.9) holds and if $\pi_{i}=\pi_{i}(0)=0$ for some $i$, then $\pi_{i}(t)=0$ for all $t>0$.

The assertion (a) follows from the formula for $\pi_{j}(t)$ given in [2]. The assertion (b) follows from (1.11).

From (a) it follows that no boundary Dirichlet conditions are needed to be given on $\partial \Pi_{n+1}$ in order to solve the variational inequality (2.1). The solution of (2.1) can be constructed as follows (cf. [2]):

For any $\delta>0, \varepsilon>0$, let

$$
\Pi_{n+1}^{o}=\left\{\pi \in \Pi_{n+1}, \pi_{i}>\delta \quad \text { for } \quad 0 \leqq i \leqq n\right\}
$$

and let $\alpha_{\varepsilon}(t)$ be a $C^{\infty}$ function in $t$ satisfying:

$$
\begin{aligned}
& \beta_{\varepsilon}^{\prime}(t) \geqq 0, \quad \beta_{\varepsilon}^{\prime \prime}(t) \geqq 0 ; \quad \beta_{\varepsilon}(t) \longrightarrow 0 \quad \text { if } \quad t<0, \quad \varepsilon \downarrow 0, \\
& \beta_{\varepsilon}(t) \longrightarrow \infty \quad \text { if } t>0, \quad \varepsilon \downarrow 0 .
\end{aligned}
$$

Consider the elliptic problem

$$
\begin{gathered}
-M u+\alpha u+\beta_{\varepsilon}(u-g)=h \text { in } \Pi_{n+1}^{\delta}, \\
u=\varphi \text { on } \partial \Pi_{n+1}^{\hat{o}}
\end{gathered}
$$

where $\varphi$ is any smooth function such that

$$
0 \leqq \varnothing \leqq g \text {. }
$$

This problem has a unique solution $u=u_{\hat{\imath}, \varepsilon}$. If $g(\pi)$ were a function in $W^{2, p}$, for any $2 \leqq p<\infty$, then one can show, by standard techniques for variational inequalities, that

$$
u_{\hat{\partial}, \varepsilon} \longrightarrow u_{\hat{o}} \text { uniformly as } \varepsilon \longrightarrow 0,
$$

where $u_{\tilde{o}}$ is the unique solution of the variational inequality

$$
\begin{aligned}
& -M u+\alpha u \leqq h \quad \text { a.e. in } \Pi_{n+1}^{o}, \\
& u \leqq g \text { in } \Pi_{n+1}^{o}, \\
& (-M u+\alpha u-h)(u-g)=0 \text { a.e. in } \Pi_{n+1}^{\delta}, \\
& u=\varphi \text { on } \partial \Pi_{n+1}^{o}, \\
& u \in W_{1, p}^{o, p}\left(\Pi_{n+1}^{o}\right), \quad u \in C\left(\bar{\Pi}_{n+1}^{\hat{o}}\right) .
\end{aligned}
$$

In the present case $g$ is not even continuously differentiable. Since however it is the minimum of linear functions in the $\pi_{i}$, it is convex. Thus, in terms of, say, $\pi_{1}, \cdots, \pi_{n}$,

$$
\left(\frac{\partial^{2} g}{\partial \pi_{i} \partial \pi_{j}}\right) \text { is negative semidefinite matrix, }
$$

where $\partial^{2} g / \partial \pi_{i} \partial \pi_{j}$ is taken in the sense of distributions. By [4] it 
follows that

$$
\left|u_{\hat{o}, \varepsilon}\right|_{W^{2}, \infty(G)} \leqq C \quad \text { if } \quad \bar{G} \subset \stackrel{\circ}{\Pi}_{n+1}^{\delta}
$$

where $C$ is a constant independent of $\delta, \varepsilon$, and then (2.5) is still valid. It follows that

$$
\left|u_{\hat{o}}\right|_{W^{2, \infty}(G)} \leqq C .
$$

We now take $\delta \rightarrow 0$ and deduce (as in [2]) that

$$
u_{\delta} \longrightarrow u \text { uniformly in compact subsets of } \stackrel{\circ}{\Pi}_{n+1}
$$

where $u$ is a solution of the variational inequality (2.1); further (by a probabilistic argument),

$$
u \text { has a continuous extension into } \Pi_{n+1},
$$

and, by (2.7),

$$
u \in W_{\mathrm{loc}}^{2, \infty}\left(\stackrel{\circ}{\Pi}_{n+1}\right) .
$$

The uniqueness of the solution $u$ subject to the smoothness conditions (2.9), (2.10) follows (as in [2]) by using Ito's formula.

We recall that $u$ can also be obtained as follows:

$$
u=\lim _{\varepsilon \rightarrow 0} \lim _{\delta \rightarrow 0} u_{\delta, \varepsilon} \text {. }
$$

Let

$$
S=\left\{\pi \in \Pi_{n+1} ; u(\pi)=g(\pi)\right\}, \quad C=\left\{\pi \in \Pi_{n+1} ; u(\pi)<g(\pi)\right\} .
$$

As in [2], $V(\pi)$ defined by (1.15) coincides in $\stackrel{\circ}{n+1}_{n+1}$ with the solution $u$ of (2.1), and an optimal stopping time $\widetilde{\tau}$ (as in (1.16)) is given by

$$
\widetilde{\tau}=\text { hitting time of } S \text { by the process } \pi(t) \text {. }
$$

Thus the optimal strategy is to continue while $\pi(t)$ is in $C$ and to stop when $\pi(t)$ hits $S$. For this reason the set $S$ is called the stopping set and the set $C$ is called the continuation set.

In the terminology of variational inequalities, $S$ is called the coincidence set, $C$ is called the noncoincidence set, and $g$ is called the obstacle. The set

$$
\Gamma=\stackrel{\circ}{\Pi}_{n+1} \cap \partial C \quad(\partial C=\text { boundary of } C)
$$

is called the free boundary.

The purpose of this paper is to study the sets $C, S$ or, equivalently, the free boundary $\Gamma$.

We shall denote by $e_{i}$ the vertex 


$$
\left(\delta_{i 0}, \delta_{i 1}, \cdots, \delta_{i n}\right)
$$

of $\Pi_{n+1}(0 \leqq i \leqq n)$.

In $\S 3$ we prove that each vertex $e_{i}$ has a $\Pi_{n+1}$-neighborhood $\widetilde{S}_{i}$ such that $\widetilde{S}_{i} \subset S$. In $\S 4$ we prove some auxiliary results needed for the following section.

In $\S 5$ we study the set

$$
S_{i}=S \cap\left\{\pi \in \Pi_{n+1} ; u(\pi)=a_{i}\left(1-\pi_{i}\right)\right\}
$$

under the assumption that

$$
q_{i, k}=0 \text { for } 0 \leqq k \leqq n .
$$

Introducing the coordinates

$$
y_{j}=\frac{\pi_{j}}{\pi_{0}} \quad(1 \leqq j \leqq n)
$$

we prove that $\Gamma_{i} \equiv \stackrel{\circ}{\Pi}_{n+1} \cap \partial S_{i}$ can be represented in the form

$$
y_{i}=\psi_{i}\left(y_{1}, \cdots, y_{i-1}, y_{i+1}, \cdots, y_{n}\right)
$$

where $\psi_{i}$ is analytic.

In $\S 6$ we specialize to the case (1.9) and prove that each $S_{i}$ is a convex set and $u(\pi)$ is a concave function.

In $\S \S 7,8$ we study the asymptotic behavior of the solution when (1.9) holds and $c \rightarrow 0$. It is shown that $\partial S_{i}$ lies within a $\delta_{1} c-$ neighborhood of $e_{i}$ and outside a $\delta_{2} c$-neighborhood of $e_{i}$. Further,

$$
E^{\pi} \widetilde{\tau} \sim\left(\sum_{i=0}^{n} \gamma_{i} \pi_{i}\right) \log \frac{1}{c}, \frac{1}{2} \gamma_{i}=\left\{\min _{k \neq i}\left|\lambda_{k}-\lambda_{i}\right|\right\}^{-1}
$$

where $\tilde{\tau}$ is the optimal stopping time, and

$$
\frac{1}{c} u(c y) \longrightarrow \widetilde{u}(y) \quad(u(y)=u(\pi))
$$

where $\tilde{u}(y)$ is the solution of a certain variational inequality; the free boundary for $\tilde{u}$ is also studied.

In $\S 9$ we consider the behavior of the solution as $c \rightarrow \infty$. The case $\alpha=0$ is considered in $\S 10$. Finally, in $\S 11$, we extend some of the results of the previous sections to the case where $w(t)$ is $k$-dimensional, for any $k$; here the methods are purely probabilistic.

We would like to thank Professors J. Sacks and A. Shiryayev for several helpful conversations.

3. $S$ contains a neighborhood of the vertices. We always denote by $u(\pi)$ the solution of (2.1) (which satisfies (2.9), (2.10); re- 
call that $u(\pi)=V(\pi)$ if $\pi \in \stackrel{\circ}{\Pi}_{n+1}$.

The operator $M u$ can be written in terms of the tangential operators of $\Pi_{n+1}$ (considered as a submanifold in $R^{n+1}$ ). Observe that on $\Pi_{n+1}$

$$
\sum_{i=0}^{n} \pi_{i}=1
$$

and, consequently, the operator

$$
\sum_{i=0}^{n} \alpha_{i} \frac{\partial}{\partial \pi_{i}}
$$

is tangential if and only if $\sum_{i=0}^{n} \alpha_{i}=0$. We introduce the tangential operators

$$
D_{0 m}=\frac{\partial}{\partial \pi_{0}}-\frac{\partial}{\partial \pi_{m}} \quad(1 \leqq m \leqq n)
$$

and the normal operator

$$
\widetilde{D}=\sum_{i=0}^{n} \frac{\partial}{\partial \pi_{i}}
$$

Substituting

$$
\frac{\partial}{\partial \pi_{i}}=-\sum_{m=1}^{n}\left(\delta_{i m}-\frac{1}{n+1}\right) D_{0 m}+\frac{1}{n+1} \widetilde{D}
$$

into $M u$ we discover that the coefficients of $\widetilde{D}^{2}, D_{0 m} \widetilde{D}$ vanish (as indeed they should) and that $M u$ takes the form

$$
\begin{gathered}
M u=\frac{1}{2} \sum_{i, j=1} \pi_{i} \pi_{j}\left(\lambda_{i}-\sum_{k=0}^{n} \lambda_{k} \pi_{k}\right) \cdot\left(\lambda_{j}-\sum_{l=0}^{n} \lambda_{l} \pi_{l}\right) D_{0 i} D_{0 j} u \\
-\sum_{j=1}^{n} \sum_{i=0}^{n} q_{i, j} \pi_{i} D_{0 j} u .
\end{gathered}
$$

Another useful coordinate system is given by (2.14), i.e.,

$$
y_{i}=\frac{\pi_{i}}{\pi_{0}} \quad(1 \leqq i \leqq n) .
$$

(The role of $\pi_{0}$ is incidental; one can similarly work with the coordinates $y_{i}=\pi_{i} / \pi_{j}, 0 \leqq i \leqq n, i \neq j$, for any fixed $j$.) It maps $\Pi_{n+1}$ onto

$$
R_{n}^{+}=\left\{y=\left(y_{1}, \cdots, y_{n}\right) ; y_{j} \geqq 0 \quad \text { for } \quad 1 \leqq j \leqq n\right\} .
$$

It is easy to compute that 


$$
\frac{\partial u}{\partial y_{i}}=\pi_{0}\left(D_{0 i}-\sum_{k=1}^{n} \pi_{k} D_{0 k}\right)
$$

and that in the $y$-coordinates $M u$ becomes (cf. [2])

$$
\begin{gathered}
L u=\frac{1}{2} \sum_{i, j=1}^{n} \mu_{i j} y_{i} y_{j} \frac{\partial^{2} u}{\partial y_{i} \partial y_{j}}+\frac{1}{Y} \sum_{i, j=1}^{n} \mu_{i j} y_{i} y_{j} \frac{\partial u}{\partial y_{j}} \\
+\sum_{j=1}^{n} \sum_{i=0}^{n}\left(q_{i, j}-q_{i, 0} y_{j}\right) y_{i} \frac{\partial u}{\partial y_{j}}
\end{gathered}
$$

where $y_{0} \equiv 1$ and

$$
\begin{aligned}
& Y=1+y_{1}+\cdots+y_{n}, \\
& \mu_{i j}=\left(\lambda_{i}-\lambda_{0}\right) \cdot\left(\lambda_{j}-\lambda_{0}\right) .
\end{aligned}
$$

We shall need the following comparison lemma:

LEMMA 3.1. Suppose that $\tilde{u}$ is a function satisfying the variational inequality (2.1) in a region $\widetilde{\Pi} \subset \Pi_{n+1}$ with $g$ replaced by $\widetilde{g}$. If

$$
\begin{aligned}
& \widetilde{g} \geqq g \text { on } \widetilde{\Pi}, \\
& \tilde{u} \geqq u \quad \text { on } \quad \text { o } \widetilde{\Pi} \cap \stackrel{\circ}{n+1}_{n} \text {, }
\end{aligned}
$$

and $\tilde{u}$ is uniformly continuous in $\widetilde{\Pi}$, then $\tilde{u} \geqq u$ in $\widetilde{\Pi}$. Similarly, if $\widetilde{g} \leqq g$ on $\widetilde{\Pi}, \tilde{u} \leqq u$ on $\partial \widetilde{\Pi} \cap \stackrel{\Pi}{n+1}_{n}$, then $\tilde{u} \leqq u$ in $\widetilde{\Pi}$.

Notice that we do not assume that $\tilde{u} \geqq u$ (or $\widetilde{u} \geqq u$ ) on $\partial \widetilde{\Pi} \cap$ $\partial \Pi_{n+1}$.

Proof. The function $\tilde{u}$ can be obtained as the limit of solutions $\tilde{u}_{\hat{o}}$ of variational inequalities in $\Pi_{n+1}^{i} \cap \widetilde{\Pi}$ (cf. (2.6)); the proof is the same as for $u$. By a standard comparison theorem for variational inequalities, $\tilde{u}_{\hat{o}} \geqq u$. Taking $\delta \rightarrow 0$, the assertion follows.

THEOREM 3.2. Assume that $c_{j}>a_{i} q_{j, i}$ for $0 \leqq j \leqq n$ and some $i$. Then there exists a $\Pi_{n+1}-$ neighborhood $\widetilde{S}_{i}$ of $e_{i}$ such that $\widetilde{S}_{i} \subset S$.

Proof. It suffices to prove the assertion for $i=0$. The proof is by comparison of $v \equiv u-g$ with a function $z$ which vanishes in an $R_{n}^{+}$-neighborhood of $y=0$. Notice that near $y=0$

$$
g=a_{0}\left(1-\pi_{0}\right)=\frac{a_{0}}{Y}\left(y_{1}+\cdots+y_{n}\right) .
$$

Since $M\left(1-\pi_{0}\right)=-\sum_{i=0}^{n} q_{20} \pi_{i}, v$ satisfies: 


$$
\begin{aligned}
& -L v+\alpha v \leqq \mu_{1}, \\
& v \leqq 0, \\
& \left(-L v+\alpha v-\mu_{1}\right) v=0
\end{aligned}
$$

a.e., if $y \in R_{n}^{+},|y| \leqq R^{*}$, where $R^{*}$ is sufficiently small and

$$
\mu_{1}=\sum_{i=0}^{n}\left(c_{i}-a_{0} q_{i 0}\right) \pi_{i}-\alpha g>c^{*}, \quad c^{*}>0 .
$$

We have to show that

$$
v(y)=0 \quad \text { if } \quad y \in R_{n}^{+}, \quad|y|<R
$$

for a sufficiently small $R$.

Let (cf. [10])

$$
\begin{gathered}
z(y)=\left\{\frac{N}{1-\theta}\left(\left(\frac{\log \frac{1}{r}}{\log \frac{1}{R}}\right)^{\theta}-\theta \frac{\log \frac{1}{r}}{\log \frac{1}{R}}\right)-N \text { if } R<r<R_{0},\right. \\
0 \text { if } \quad r<R
\end{gathered}
$$

where $0<\theta<1, N>0, r=|y|$. We compute that $(\partial z / \partial r)<0$ if $R<r<R_{0}$, so that $z<0$. Also

$$
z=\frac{\partial z}{\partial r}=0 \text { if } r=R
$$

If we show that

$$
\gamma \equiv-L z+\alpha z \quad \text { satisfies } \quad \gamma \leqq \mu_{1} \quad\left(R<r<R_{0}\right)
$$

and if also

$$
z\left(R_{0}\right) \leqq-K \text { where } K=\sup v
$$

then, by Lemma $3.1, z \leqq v$ if $0<r<R_{0}$. This implies that $0 \leqq v$ if $0<r<R$, and (3.11) follows.

To establish (3.12), (3.13) we compute

$$
\begin{aligned}
& \left|L\left(\log \frac{1}{r}\right)\right| \leqq C \\
& \left|L\left(\log \frac{1}{r}\right)^{\theta}\right| \leqq \frac{C}{\left(\log \frac{1}{r}\right)^{1-\theta}} .
\end{aligned}
$$

It follows that 


$$
\gamma=-L z+\alpha z \leqq \frac{C N}{\log \frac{1}{R}}+\alpha z \leqq \frac{C N}{\log \frac{1}{R}}
$$

Thus it suffices to satisfy (using (3.10))

$$
\frac{C N}{\log \frac{1}{R_{0}}}=c_{*}
$$

and

$$
\frac{N}{1-\theta}\left(M^{\theta}-\theta M\right)-N \leqq-K \quad\left(M=\frac{\log \frac{1}{R_{0}}}{\log \frac{1}{R}}<1\right)
$$

Choosing $M$ sufficiently small so that

$$
\frac{M^{\theta}-\theta M}{1-\theta} \leqq \frac{1}{2}
$$

and taking $N>2 K,(3.15)$ follows. Defining $R_{0}$ by (3.14), the proof is complete.

\section{Auxiliary results.}

Definition. A point $\pi \in \grave{\Pi}_{n+1}$ is said to belong to the ridge $R$ of the obstacle $g$ if $g$ is not $W^{2, \infty}$ in any neighborhood of $\pi$.

Thus, $\pi=\left(\pi_{0}, \cdots, \pi_{n}\right) \in R$ if and only if

$$
a_{i}\left(1-\pi_{i}\right)=a_{j}\left(1-\pi_{j}\right) \text { for some } i \neq j .
$$

The above definition is analogous to the definition used in elasticplastic torsion problems [6] where $g$ is the distance function from the boundary of the domain.

THEOREM 4.1. The ridge is contained in $C$.

Proof. Suppose $\tilde{\pi}=\left(\tilde{\pi}_{0}, \cdots, \tilde{\pi}_{n}\right) \in R$ and, say,

$$
a_{1}\left(1-\tilde{\pi}_{1}\right)=a_{2}\left(1-\tilde{\pi}_{2}\right) \text {. }
$$

If $\tilde{\pi} \in S$ then

$$
\begin{aligned}
& \nabla\left(u-a_{1}\left(1-\pi_{1}\right)\right)=0, \\
& \nabla\left(u-a_{2}\left(1-\pi_{2}\right)\right)=0
\end{aligned}
$$

at $\tilde{\pi}$, since $u-a_{i}\left(1-\pi_{i}\right) \leqq 0$ in $\Pi_{n+1}$ and $u(\tilde{\pi})-a_{i}\left(1-\tilde{\pi}_{i}\right)=0, i=$ 
1, 2. Thus

$$
\nabla\left(a_{1} \pi_{1}-a_{2} \pi_{2}\right)=0 \text { at } \tilde{\pi}
$$

But

$$
\begin{aligned}
\left(\frac{\partial}{\partial y_{1}}-\frac{\partial}{\partial y_{2}}\right)\left(a_{1} \pi_{1}-a_{2} \pi_{2}\right) & =\left(\frac{\partial}{\partial y_{1}}-\frac{\partial}{\partial y_{2}}\right) \frac{a_{1} y_{1}-a_{2} y_{2}}{Y} \\
=\frac{a_{1}+a_{2}}{Y} & >0 ;
\end{aligned}
$$

a contradiction.

Lemma 4.2. Assume that $q_{i, k}=0$ for $0 \leqq k \leqq n$ and some $i \geqq 1$. Then

$$
\frac{\partial}{\partial y_{i}}\left[Y\left(u-a_{i}\left(1-\pi_{i}\right)\right)\right] \geqq 0
$$

Proof. It suffices to prove (4.1) for $i=1$. In $\S 2$ we may replace $\Pi_{n+1}^{\delta}$ by any other sequence of domains which increase to $\Pi_{n+1}$ and the boundary values $\varphi$ on $\partial \Pi_{n+1}^{\delta}$ by any continuous function $\varphi$ satisfying $0 \leqq \varphi \leqq g$. We shall choose $\Pi_{n+1}^{\delta}$ so that in the $y$-coordinates it becomes

$$
G_{\delta}=\left\{y ; \delta<y_{i}<\frac{1}{\delta} \text { for } 1 \leqq i \leqq n\right\} .
$$

Let

$$
\begin{aligned}
& v=u_{\delta}-a_{1}\left(1-\pi_{1}\right) \\
& z=Y v
\end{aligned}
$$

and choose $\varphi$ as follows:

$$
u_{\delta}=0 \text { on } y_{1}=\delta \text {; }
$$

$Y u_{\delta}$, on each face $y_{i}=\delta$ or $y_{i}=1 / \delta(2 \leqq i \leqq n)$, is a

(4.3) monotone increasing function of $y_{1}$ such that $Y u_{\delta} \leqq Y g$, and $Y u_{\hat{\delta}}=0$ at $y_{1}=\delta, Y u_{\delta}=Y g$ at $y_{1}=1 / \delta ; Y u_{\delta}=$ $Y g$ on $y_{1}=1 / \delta$.

Then, on $y_{1}=\delta$

$$
\left.\frac{\partial}{\partial y_{1}}\left(Y u_{\hat{o}}\right) \geqq 0 \text { (since } u_{\hat{o}}=0 \text { on } y_{1}=\delta, u_{\delta} \geqq 0 \text { elsewhere }\right) .
$$




$$
a_{1} Y\left(1-\pi_{1}\right)=a_{1}\left(1+y_{2}+\cdots+y_{n}\right)
$$

so that

$$
-\frac{\partial}{\partial y_{1}}\left(a_{1} Y\left(1-\pi_{1}\right)\right)=0
$$

Consequently

$$
\frac{\partial z}{\partial y_{1}} \geqq 0 \quad \text { on } \quad y_{1}=\delta .
$$

On $y_{i}=\delta$ or $y_{i}=1 / \delta(2 \leqq i \leqq n)$ we have, by (4.3),

$$
\frac{\partial}{\partial y_{1}}\left(Y u_{\hat{\nu}}\right) \geqq 0 \text {. }
$$

Using (4.4) we again get

$$
\frac{\partial z}{\partial y_{1}} \geqq 0 \quad \text { on } \quad y_{\imath}=\delta \quad \text { or } \quad y_{i}=\frac{1}{\delta} \quad(2 \leqq i \leqq n) .
$$

On $y_{1}=1 / \delta, z=0$ by (4.3). Since $z \leqq 0$ elsewhere, we obtain

$$
\frac{\partial z}{\partial y_{1}} \geqq 0 \quad \text { on } \quad y_{1}=\frac{1}{\delta} .
$$

Denote by $C_{\hat{o}}$ the set where $u_{\hat{o}}<g$. Then, in $C_{\hat{o}}$,

$$
M v-\alpha v=-\sum_{i=0}^{n}\left(c_{i}-a_{i} q_{i, 1}\right) \pi_{i}+\alpha a_{1}\left(1-\pi_{1}\right) .
$$

Recalling that $L v=M v$ where $L$ is defined by (3.5), and substituting

$$
\begin{gathered}
\frac{\partial v}{\partial y_{i}}=\frac{1}{Y} \frac{\partial z}{\partial y_{i}}-\frac{1}{Y^{2}} z, \\
\frac{\partial^{2} v}{\partial y_{i} \partial y_{j}}=\frac{1}{Y} \frac{\partial^{2} z}{\partial y_{i} \partial y_{j}}-\frac{1}{Y^{2}} \frac{\partial z}{\partial y_{i}}-\frac{1}{Y^{2}} \frac{\partial z}{\partial y_{j}}+\frac{2}{Y^{2}} z,
\end{gathered}
$$

we find that

$$
L_{0} z-\alpha z=-\sum_{i=0}^{n}\left(c_{i}-a_{1} q_{i, 1}\right) y_{i}+\alpha a_{1}\left(Y-y_{1}\right)
$$

where

$$
\begin{gathered}
L_{0} z \equiv \frac{1}{2} \sum_{i, j=1}^{n} \mu_{i j} y_{i} y_{j} \frac{\partial^{2} z}{\partial y_{i} \partial y_{j}}+\sum_{j=1}^{n} \sum_{i=0}^{n}\left(q_{i, j}-q_{i, 0} y_{j}\right) y_{i} \frac{\partial z}{\partial y_{j}} \\
+\sum_{i=0}^{n} q_{i, 0} y_{i} z
\end{gathered}
$$


Differentiating (4.8) with respect to $y_{1}$, we obtain the following equation for $w=\partial z / \partial y_{1}$ :

$$
\begin{gathered}
L_{0} w+\sum_{i=1}^{n} \mu_{i 1} y_{i} \frac{\partial w}{\partial y_{i}}+\sum_{i=0}^{n} \sum_{j=1}^{n}\left(q_{i, j}-q_{i, 0} y_{j}\right) y_{i} \frac{\partial w}{\partial y_{j}}-\alpha w \\
=-\left(c_{1}-a_{1} q_{1,1}\right)=-c_{1} .
\end{gathered}
$$

From the maximum principle it then follows that $w>0$ in $C_{\delta}$ provided $w \geqq 0$ on $\partial C_{\hat{j}}$. In view of (4.5)-(4.7), $w \geqq 0$ on $\partial C_{\delta} \cap G_{\dot{o}}$. We next show that

$$
w(\widetilde{y}) \geqq 0 \quad \text { if } \quad \widetilde{y} \in \partial C_{\hat{o}} \cap\left(\operatorname{int} G_{\hat{o}}\right) .
$$

Indeed, since $\widetilde{y} \in \partial C_{\delta} \cap\left(\operatorname{int} G_{\delta}\right)$,

$$
u_{\hat{o}}=a_{i}\left(1-\pi_{i}\right), \quad \nabla u_{\hat{o}}=\nabla\left(a_{i}\left(1-\pi_{i}\right)\right) \text { at } \widetilde{y},
$$

for some $i$ for which $g=a_{i}\left(1-\pi_{i}\right)$ at $\widetilde{y}$. Writing

$$
\begin{gathered}
w=\frac{\partial z}{\partial y_{1}}=\frac{\partial}{\partial y_{1}}\left[Y u_{\delta}-a_{i} Y\left(1-\pi_{i}\right)\right]+\frac{\partial}{\partial y_{1}}\left[a_{i} Y\left(1-\pi_{i}\right)\right] \\
-\frac{\partial}{\partial y_{1}}\left[a_{1} Y\left(1-\pi_{1}\right)\right]
\end{gathered}
$$

we note that the first term on the right hand side vanishes by (4.12), the third one vanishes by (4.4), and the middle one is equal to

$$
\begin{aligned}
a_{i} \frac{\partial}{\partial y_{1}}\left(Y-y_{i}\right) & =a_{i} \text { if } i \neq 1, \\
& =0 \text { if } i=1,
\end{aligned}
$$

we conclude that $w(\widetilde{y}) \geqq 0$.

It follows that

$$
\frac{\partial}{\partial y_{1}}\left[Y\left(u_{\delta}-a_{1}\left(1-\pi_{1}\right)\right)\right] \geqq 0 \text { in } C_{\delta} \text {; hence also in } G_{\delta} .
$$

Taking $\delta \rightarrow 0$, the assertion of the lemma follows.

REMARK. Recalling (3.4), we can rewrite the assertion of Lemma 4.2 as follows:

$$
\left(D_{0 i}-\sum_{k=1}^{n} \pi_{k} D_{0 k}\right)\left[Y\left(u-a_{i}\left(1-\pi_{i}\right)\right)\right] \geqq 0 .
$$

If we replace the role of $e_{0}$ by another vertex, say $e_{n}$, the corresponding differential inequality 


$$
\left(D_{n i}-\sum_{k=0}^{n-1} \pi_{k} D_{n k}\right)\left[Y\left(u-a_{i}\left(1-\pi_{i}\right)\right)\right] \geqq 0
$$

(where $D_{n j}=\partial / \partial \pi_{n}-\partial / \partial \pi_{j}$ ) coincides with (4.13); thus we do not get any new inequality.

5. The free boundary is analytic. We continue to use the $y$ coordinates (3.3).

Denote by $G_{i}(0 \leqq i \leqq n)$ the open components of $\Pi_{n+1} \backslash R$ with $\partial G_{i} \ni e_{i}$ and set

$$
S_{i}=S \cap G_{i}, \quad C_{i}=C \cap G_{i},
$$

the definition of $S_{i}$ is the same as in (2.13). Denote by $\breve{G}_{i}, \widetilde{S}_{i}, \widetilde{C}_{i}$ the images of $G_{i}, S_{i}, C_{i}$ respectively in the $y$-coordinates. Denote by $\widetilde{R}$ the image of the ridge $R$ in the $y$-coordinates. It is easy to check that if $\widetilde{y}=\left(\widetilde{y}_{0}, \cdots, \widetilde{y}_{n}\right) \in \widetilde{G}_{i}$ then there is a line segment

$$
\gamma=\left\{y ; y_{j}=\widetilde{y}_{j} \quad \text { if } \quad j \neq i, \widetilde{y}_{i}-\beta<y_{i} \leqq \widetilde{y}_{i}\right\} \quad(\beta>0)
$$

which belongs to $\widetilde{G}_{i}$ and its left end point lies on $\widetilde{R}$.

Suppose now that

$$
q_{i, k}=0 \text { for } 0 \leqq k \leqq n \text { and some } i \geqq 1 .
$$

By Lemma 4.2 we then deduce that if

$$
u(\widetilde{y})-g(\widetilde{y})<0, \quad \widetilde{y} \in \widetilde{G}_{i}
$$

then

$$
u\left(y^{*}\right)-g\left(y^{*}\right)<0 \text { for any } y^{*} \in \gamma .
$$

Thus the open set $C_{i}$ is connected to $R$. Since $R$ belongs to $C$, by Theorem 4.1 it follows that $C_{i}$ is connected.

The previous argument involving (5.2), (5.3) shows also that there exists a function $\psi_{i}\left(y_{1}, \cdots, y_{i-1}, y_{i+1}, \cdots, y_{n}\right)$ such that

$$
\widetilde{C}_{i}=\left\{y \in \breve{G}_{i} ; y_{i}<\psi_{i}\left(y_{1}, \cdots, y_{i-1}, y_{i+1}, \cdots, y_{n}\right)\right\} \text {. }
$$

We can thus state:

THEOREM 5.1. If (5.2) holds then $C_{i}$ is connected and it is a subgraph in the sense of (5.5).

We next prove that $\psi_{i}$ is analytic:

THEOREM 5.2. If (5.2) holds then $\psi_{i}$ is analytic. More precisely, if 


$$
\begin{aligned}
& \widetilde{y}_{i}=\psi_{i}\left(\widetilde{y}_{1}, \cdots, \widetilde{y}_{i-1}, \widetilde{y}_{i+1}, \cdots, \widetilde{y}_{n}\right), \\
& \left(\widetilde{y}_{1}, \cdots, \widetilde{y}_{n}\right) \in \operatorname{int} R_{n}^{+}
\end{aligned}
$$

then $\psi_{i}$ is analytic at $\left(\widetilde{y}_{1}, \cdots, \widetilde{y}_{i-1}, \widetilde{y}_{i+1}, \cdots, \widetilde{y}_{n}\right)$. Thus the free boundary in the interior of $G_{i}$ is analytic.

The proof of Theorem 5.2 given below is based on a method of Alt [1].

Proof. It suffices to prove the theorem for $i=n$. Let $y^{\prime}=$ $\left(y_{1}, \cdots, y_{n-1}\right)$ and consider the function

$$
\zeta=a_{n}\left(1-\pi_{n}\right)-u
$$

in

$$
D_{\rho_{0}}=\left\{\beta<y_{n}<\psi_{n}\left(y^{\prime}\right),\left|y^{\prime}-y_{0}^{\prime}\right|<\rho_{0}\right\} .
$$

Here $y_{0}^{\prime}$ is a fixed point with positive coordinates, $\beta<\psi_{n}\left(y_{0}^{\prime}\right)$, and $\psi_{n}\left(y_{0}^{\prime}\right)-\beta, \rho_{0}$ are sufficiently small so that $D_{\rho_{0}}$ is contained in $\widetilde{C}_{n}$. We have $\zeta>0$ in $D_{\rho_{0}}, \zeta=0$ on $y_{n}=\psi_{n}\left(y^{\prime}\right)$. By Lemma 4.2,

$$
\frac{\partial \zeta}{\partial y_{n}}<0 \text { in } D_{\rho_{0}} \text {. }
$$

Consider the function

$$
\eta=\sum_{k=1}^{n-1} \alpha_{k} \frac{\partial \zeta}{\partial y_{k}}-A \frac{\partial \zeta}{\partial y_{n}}
$$

in $D_{\rho_{0}}$, where $\sum \alpha_{k}^{2} \leqq 1$ and $A$ is a sufficiently large positive constant to be determined later on. We have (cf. (4.8))

$$
L_{0} \zeta-\alpha \zeta=\sum_{i=0}^{n}\left(c_{i}-a_{n} q_{i, n}\right) y_{i}-\alpha a_{n}\left(Y-y_{n}\right) \equiv \tilde{k}
$$

Differentiating with respect to $y_{k}$, first when $k=n$ and then when $1 \leqq k \leqq n-1$, we get

$$
\begin{aligned}
& L_{0}\left(\frac{\partial \zeta}{\partial y_{n}}\right)+\sum_{i=1}^{n} \mu_{i n} y_{i} \frac{\partial}{\partial y_{i}}\left(\frac{\partial \zeta}{\partial y_{n}}\right)-\alpha \frac{\partial \zeta}{\partial y_{n}}=\frac{\partial \tilde{k}}{\partial y_{n}}=c_{n}, \\
& L_{0}\left(\frac{\partial \zeta}{\partial y_{k}}\right)+\sum_{i=1}^{n} \mu_{i n} y_{i} \frac{\partial}{\partial y_{i}}\left(\frac{\partial \zeta}{\partial y_{k}}\right)-\alpha \frac{\partial \zeta}{\partial y_{k}}=\frac{\partial \tilde{k}}{\partial y_{k}}+M_{k}
\end{aligned}
$$

where

$$
M_{k}=\sum_{i=1}^{n}\left(\mu_{i n}-\mu_{i k}\right) y_{i} \frac{\partial^{2} \zeta}{\partial y_{i} \partial y_{k}}+\sum_{i=1}^{n} \lambda_{k i} \frac{\partial \zeta}{\partial y_{i}}+\tilde{\lambda}_{k} \zeta
$$

where $\lambda_{i k}, \tilde{\lambda}_{k}$ are linear functions, and 


$$
\frac{\partial \widetilde{k}}{\partial y_{k}}=c_{k}-a_{n} q_{k, n}-\alpha a_{n} \text {. }
$$

Since $\zeta \in W_{\text {loc }}^{2, \infty}, M_{k}$ is bounded and consequently,

$$
\widetilde{L} \eta \equiv L_{0} \eta+\sum_{i=1}^{n} \mu_{i n} y_{i} \frac{\partial \eta}{\partial y_{i}}-\alpha \eta=-A c_{n}+B
$$

where $B$ is bounded indepedenntly of the $\alpha_{i}$ and $A$.

We choose $A$ sufficiently large so that

$$
\tilde{L} \eta \leqq-1 \text { in } D_{\rho_{0}} \text {. }
$$

Now let $\rho$ be any number $<\rho_{0}$ (for instance $\rho=\rho_{0} / 2$ ) and define

$$
D_{\rho}=\left\{\beta<y_{n}<\psi_{n}\left(y^{\prime}\right),\left|y^{\prime}-y_{0}^{\prime}\right|<\rho\right\} .
$$

Denote by $\partial D_{\rho}$ the boundary of $D_{\rho}$ and set

$$
\begin{aligned}
& \Gamma_{\rho, \sigma}=\left\{y \in \partial D_{\rho} ; y_{n}<\psi_{n}\left(y^{\prime}\right)-\sigma\right\}, \\
& \widetilde{\Gamma}_{\rho, \sigma}=\left\{y \in \partial D_{\rho} ; \psi_{n}\left(y^{\prime}\right)-\sigma<y_{n}<\psi_{n}\left(y^{\prime}\right)\right\} .
\end{aligned}
$$

Define $\partial D_{\rho_{0}}, \Gamma_{\rho_{0}, \sigma}, \Gamma_{\rho_{0}, \sigma}$ similarly with respect to $D_{\rho_{0}}$.

For any sufficiently small $\sigma>0$ we have, by (5.6),

$$
\eta>0 \text { in } \Gamma_{\rho, \sigma} \cup \Gamma_{\rho, \sigma}
$$

provided $A=A(\sigma)$ is sufficiently large. We claim that if $\sigma$ is sufficiently small depending on $\rho, \rho_{0}$ then

$$
\eta \geqq 0 \text { on } \Gamma_{\rho, \sigma} \text {. }
$$

Indeed, suppose (5.11) is not true. Then there exists a point $y^{*} \epsilon$ $\widetilde{\Gamma}_{\rho, \sigma}$ such that $\eta\left(y^{*}\right)<0$.

Consider the function

$$
\tilde{\eta}=\eta+\gamma\left|y-y^{*}\right|^{2} \quad(\gamma>0) .
$$

If $\gamma$ is sufficiently small then $\tilde{L} \tilde{\eta}<0$. Therefore, $\tilde{\eta}$ cannot take negative minimum in $D_{\rho_{0}}$. But since $\tilde{\eta}\left(y^{*}\right)<0, \tilde{\eta}>0$ on $\Gamma_{\rho_{0}, \sigma}$ (by (5.10)) and on $y_{n}=\psi_{n}\left(y^{\prime}\right)$, there must exist a point $\hat{y} \in \widetilde{\Gamma}_{\rho_{0}, \sigma}$ such that $\tilde{\eta}(\hat{y})<0$. Thus

$$
\sum_{k=1}^{n-1} \alpha_{k} \frac{\partial \zeta}{\partial y_{k}}-A \frac{\partial \zeta}{\partial y_{n}}+\gamma\left|\hat{y}-y^{*}\right|^{2}<0
$$

Recalling that $-A\left(\partial \zeta / \partial y_{n}\right)>0$, and that

$$
\frac{\partial \zeta}{\partial y_{k}}=0(\sigma) \text { on } \quad \widetilde{\Gamma}_{\rho_{0}, \sigma}
$$


(since $\zeta \in W_{\mathrm{loc}}^{2, \infty}$ and $\nabla \zeta=0$ on $y_{n}=\psi_{n}\left(y^{\prime}\right)$ ), we deduce that

$$
\left(\rho_{0}-\rho\right)^{2} \leqq C \sigma
$$

where $C$ is a constant independent of $\rho_{0}, \rho, \sigma, A$. Consequently, if $\sigma$ is sufficiently small so that (5.12) is not true then the inequality (5.11) is valid. It follows that $\eta \geqq 0$ on $\partial D_{\rho}$. Applying the maximum principle we conclude that $\eta>0$ in $D_{\rho}$, i.e.,

$$
\sum_{k=1}^{n-1} \alpha_{k} \frac{\partial \zeta}{\partial y_{k}}-A \frac{\partial \zeta}{\partial y_{n}}>0 \text { in } D_{\rho} .
$$

Denote by $K$ the cone

$$
\left\{y ; y_{n}<-\frac{\left|y^{\prime}\right|}{A}\right\} .
$$

The inequality (5.13) implies that if

$$
\widetilde{y}=\left(\widetilde{y}^{\prime}, \widetilde{y}_{n}\right), \quad \widetilde{y}_{n}=\psi_{n}\left(\widetilde{y}^{\prime}\right)
$$

then $\zeta>0$ in the cone $K+\widetilde{y}$. Thus, if $\widehat{y}_{n}=\dot{\psi}_{n}\left(\hat{y}^{\prime}\right)$ then

$$
\hat{y} \notin K+\widehat{y},
$$

i.e.,

$$
\hat{y}_{n}>\tilde{y}_{n}-\frac{\left|\hat{y}^{\prime}-\tilde{y}^{\prime}\right|}{A},
$$

or equivalently,

$$
\psi_{n}\left(\hat{y}^{\prime}\right)>\psi_{m}\left(\widetilde{\eta}^{\prime}\right)-\frac{\left|\hat{y}^{\prime}-\widetilde{y}^{\prime}\right|}{A} .
$$

Interchanging $\hat{y}$ with $\widetilde{y}$ we deduce that

$$
\left|\psi_{n}\left(\hat{y}^{\prime}\right)-\psi_{n}\left(\widetilde{y}^{\prime}\right)\right| \leqq \frac{\left|\hat{y}^{\prime}-\widetilde{y}^{\prime}\right|}{A},
$$

that is, $\psi_{n}$ is Lipschitz continuous.

By a general result of Caffarelli [5] it then follows that $\psi_{n}$ is a $C^{1}$ function and then (by Kinderlehrer and Nirenberg [12]) also analytic.

REMARK. It is clear that Theorems 5.1, 5.2 extend to the case where (5.2) holds with $i=0$. Instead of using the coordinate transformation (3.3), we take $y_{j}=\pi_{j} / \pi_{j_{0}}$ for $0 \leqq j \leqq n, j \neq j_{0}$ for any $j_{0}, j_{0} \neq 0$.

6. The special case (1.9). In this section we obtain additional 
results in the special case when (1.9) holds. For any $0 \leqq j \leqq n$, let

$$
\pi_{j}^{\prime}=\left(\pi_{0}, \pi_{1}, \cdots, \pi_{j-1}, \pi_{j+1}, \cdots, \pi_{n}\right)
$$

and denote by $\tilde{u}_{j}\left(\pi_{j}^{\prime}\right)$ the solution of $(2.1)$ corresponding to the problem with $n$ hypotheses $H_{i}, 0 \leqq i \leqq n, i \neq j$.

TheOREM 6.1. Suppose $q_{i, j}=0$ for $0 \leqq i, j \leqq n$. If $\pi_{j}>0$, $\pi_{j} \downarrow 0$ then

$$
u(\pi) \longrightarrow \widetilde{u}_{j}\left(\pi_{j}^{\prime}\right)
$$

REMARK. Recall that boundary values for $u$ were not prescribed (on $\left.\partial \Pi_{n+1}\right)$; in fact, in $\Pi_{n+1}$,

$$
u(\pi)=\inf _{\tau} E^{\pi}\left[\int_{0}^{\tau} e^{-\alpha t} h(\pi(t)) d t+e^{-\alpha \tau} g(\pi(\tau))\right] \equiv V(\pi)
$$

and, as shown in [2], the middle term is uniformly continuous in $\stackrel{\Pi}{n+1}_{n}$. This implies that $u$ has a continuous extension into $\partial \Pi_{n+1}$, which is denoted again by $u$. What we have to prove is that this extension, when restricted to $\pi_{j}=0$, coincides with $\tilde{u}_{j}\left(\pi_{j}^{\prime}\right)$.

Proof. If suffices to consider the case $j=n$. Let $\pi^{\prime}=\pi_{n}^{\prime}$ and $\widetilde{u}\left(\pi^{\prime}\right)=\tilde{u}_{n}\left(\pi_{n}^{\prime}\right)$. We denote by $\tau_{\delta}$ the exit time of $\pi(t)$ from $\Pi_{n+1}^{\delta}$. We shall compare the cost functions

$$
\begin{aligned}
J_{\pi}(\tau)=E^{\pi}\left[\int_{0}^{\tau \wedge \tau_{\delta}} c e^{-\alpha t} d t+e^{-\alpha \tau} g(\pi(\tau)) I_{\tau \leqq \tau_{\delta}}\right. \\
\\
\left.+e^{-\alpha \tau_{\delta}} \varphi\left(\pi\left(\tau_{\delta}\right)\right) I_{\tau \geqq \tau_{\delta}}\right] \\
J_{\pi^{\prime}}(\tau)=E^{\pi^{\prime}}\left[\int_{0}^{\tau} c e^{-\alpha t} d t+e^{-\alpha \tau} g_{1}(\tilde{\pi})\right]
\end{aligned}
$$

where $\tilde{\pi}(t)=\left(\tilde{\pi}_{0}(t), \cdots, \tilde{\pi}_{n-1}(t), \tilde{\pi}_{n}(t)\right)$ is the process $\pi(t)$ with $\tilde{\pi}(0)=$ $\left(\pi^{\prime}, 0\right)$ and

$$
g_{1}(\pi)=\min _{0 \leqq i \leqq n-1}\left\{a_{i}\left(1-\pi_{i}\right)\right\}
$$

Recall [2] that

$$
\begin{aligned}
& u_{\delta}(\pi)=\inf _{\tau} J_{\pi}(\tau) \\
& \tilde{u}\left(\pi^{\prime}\right)=\inf _{\tau} J_{\pi^{\prime}}(\tau)
\end{aligned}
$$

where $\tau$ varies over all $\mathscr{F}_{t}$ stopping times.

By Lemma 2.1, $\tilde{\pi}_{n}(t) \equiv 0$.

In what follows we shall use a model of the Markov process associated with $\pi(t)$ in which the probability is fixed, say $P$, and the initial condition $\pi(0)=\pi$ varies; for each $\pi, \pi(t)$ is the solution 
of the stochastic differential system associated with the generator $M$, and the initial condition $\pi(0)=\pi$. Working with this model, we can replace $E^{\pi}, E^{\pi^{\prime}}$ by $E$, and we shall compare $J_{\pi}(\tau), J_{\pi^{\prime}}(\tau)$ with the same $\tau$. We have (see, for instance, [8]), for any $T>0$,

$$
E\left[\sup _{0 \leqq t \leqq T}|\pi(t)-\tilde{\pi}(t)|^{2}\right] \leqq C_{T} \pi_{n}^{2}, \quad C_{T} \text { constant } .
$$

By Lemma 2.1, for any $\eta>0$,

$$
P\left[\tau_{\dot{o}}<T\right]<\eta \quad \text { if } \pi(0)=\left(\pi_{0}, \cdots, \pi_{n}\right), \quad \pi_{n}>0
$$

provided $\delta$ is sufficiently small (depending on $\eta, \pi_{n}$ ).

Next, by Lemma 2.1 and (6.3),

$$
E\left|g(\pi(t))-g_{1}(\tilde{\pi}(t))\right| \leqq C_{T} \pi_{n} \quad \text { if } \quad 0<t \leqq T .
$$

Using (6.3)-(6.5) we find that

$$
\left|J_{\pi}(\tau)-J_{\pi^{\prime}}(\tau)\right| \leqq C C_{T} \pi_{n}+C e^{-\alpha T}+C \eta
$$

if $\delta$ is sufficiently small, depending on $\eta$. Recalling (6.2) we get

$$
\left|u(\pi)-\widetilde{u}\left(\pi^{\prime}\right)\right| \leqq C C_{T} \pi_{n}+C e^{-\alpha T}+C \eta .
$$

Taking $\delta \rightarrow 0$ and ssing (2.8), we obtain

$$
\left|u(\pi)-\widetilde{u}\left(\pi^{\prime}\right)\right| \leqq C C_{T} \pi_{n}+C e^{-\alpha T}+C \eta .
$$

Taking $\pi_{n} \rightarrow 0$ we conclude that

$$
\lim \sup \left|u(\pi)-\widetilde{u}\left(\pi^{\prime}\right)\right| \leqq C e^{-\alpha T}+C \eta .
$$

Taking $\eta \rightarrow 0, T \rightarrow \infty$ the assertion (6.1) follows.

THEOREM 6.2. If (1.9) holds then each set $S_{\imath}$ is a convex set and the function $u(\pi)$ is concave.

The proof is analogous to that for the discrete case [16].

Proof. Let $\pi^{1}, \pi^{2}$ belong to $\stackrel{\circ}{\Pi}_{n+1}$ and set

$$
\tilde{\pi}=\lambda \pi^{1}+(1-\lambda) \pi^{2} \quad(0<\lambda<1) .
$$

We can write (1.6) in the form

$$
J_{i=}(\hat{\delta})=\sum_{i=0}^{n} \pi_{i} E^{i}\left[c \int_{0}^{\tau} e^{-\alpha t} d t+e^{-\alpha \tau} W(\theta(\omega), d(\omega))\right],
$$

where $E^{i}=E^{\pi}$ for $\pi=e_{i}$. Writing this relation for a specific $\delta=$ $(\tau(\omega), d(\omega))$ and $\pi=\pi^{1}, \pi=\pi^{2}$, and multiplying the first relation by $\lambda$ and the second one by $(1-\lambda)$ we obtain, upon adding these re- 
lations,

$$
\begin{gathered}
\lambda J_{\pi^{1}}(\delta)+(1-\lambda) J_{\pi^{2}}(\delta)=\sum_{i=0}^{n}\left(\lambda \pi_{i}^{1}+(1-\lambda) \pi_{i}^{2}\right) E^{i}[\cdots] \\
=\sum_{i=0}^{n} \tilde{\pi}_{i} E^{i}[\cdots]=E^{\tilde{\pi}}[\cdots]=J_{\tilde{\pi}}(\delta) ;
\end{gathered}
$$

here $\pi_{i}^{1}, \pi_{i}^{2}, \tilde{\pi}_{i}$ are the $i$ th coodinates of $\pi^{1}, \pi^{2}, \tilde{\pi}$ respectively and the expression $[\cdots]$ is the same as in (6.6).

Suppose now that $\pi^{1}$ and $\pi^{2}$ belong to $S_{i}$. Then

$$
a_{i}\left(1-\pi_{i}^{1}\right) \leqq J_{\pi^{1}}(\delta), \quad a_{\imath}\left(1-\pi_{i}^{2}\right) \leqq J_{\pi^{2}}(\delta) .
$$

It follows from (6.7) that

$$
a_{i}\left(1-\tilde{\pi}_{i}\right) \leqq J_{\tilde{\pi}}(\delta) .
$$

Thus $\tilde{\pi} \in S_{\imath}$ and, consequently, $S_{i}$ is a convex set.

Next, (6.7) gives

$$
\inf _{\delta} J_{\tilde{\tilde{\pi}}}(\delta) \geqq \lambda \inf _{\tilde{\delta}} J_{\pi^{1}}(\delta)+(1-\lambda) \inf _{\delta} J_{\tilde{\pi}^{2}}(\delta),
$$

i.e.,

$$
u\left(\lambda \pi^{1}+(1-\lambda) \pi^{2}\right)=u(\tilde{\pi}) \geqq \lambda u\left(\pi^{1}\right)+(1-\lambda) u\left(\pi^{2}\right),
$$

so that $u(\pi)$ is concave.

REMARK 1. From Theorems 6.2, 5.2, 3.2 we deduce that each $S_{i}$ is a convex domain containing a $\Pi_{n+1}$-neighborhood of $e_{i}$ and $\partial S_{i} \cap$ $\stackrel{\circ}{n+1}_{n}$ is an analytic manifold.

REMARK 2. For any numbers $\alpha_{i, k}(0 \leqq i \leqq n, 1 \leqq k \leqq l)$ the equations

$$
\sum_{i=1}^{n} \alpha_{i, k} y_{i}=\alpha_{0, k}\left(1 \leqq k \leqq l, y_{i}=\frac{\pi_{i}}{\pi_{0}}\right)
$$

hold if and only if

$$
\left.\sum_{i=1}^{n} \alpha_{i, k} \pi_{i}-\alpha_{0, k} \pi_{0}=0 \quad 1 \leqq k \leqq l\right) .
$$

Since also $\sum_{i=0}^{n} \pi_{i}=1$, it follows that the mapping (3.3) maps planes onto planes and lines onto lines. It also maps segments onto segments. It follows, in particular, that

(3.3) maps convex sets onto convex sets .

Consequently, by Theorem 6.2 , the image ${\widetilde{S_{i}}}_{i}(1 \leqq i \leqq n)$ in the $y$-space of the coincidence set $S_{i}$ (in the $\pi$-space) is a convex set. 
7. Asymptotic estimates for $c \rightarrow 0$. For any $\gamma>0$ set

$$
N_{\gamma}^{i}=\left\{\pi \in \Pi_{n+1} ; 1-\gamma \leqq \pi_{i}<1\right\}, \quad N_{\gamma}=\bigcup_{i=0}^{n} N_{\gamma}^{i} .
$$

THEOREM 7.1. Assume that (1.9) holds. Then there exist positive constants $\delta_{1}, \delta_{2}$ independent of $c, \alpha$, such that for all $c$ sufficiently small,

$$
N_{\delta_{1} c} \subset S \subset N_{\delta_{2} c}
$$

Proof. Set

$$
r=\sum_{i=1}^{n} \pi_{i} .
$$

Using (3.2) and the relation $\sum_{j=1}^{n} q_{i, j}=-q_{i, 0}$ we find that (for general $q_{i, j}$ )

$$
M(\log r)=-\frac{1}{2 r^{2}}\left|\sum_{i=1}^{n} \sum_{k=0}^{n}\left(\lambda_{i}-\lambda_{k}\right) \pi_{i} \pi_{k}\right|^{2}-\frac{1}{r} \sum_{i=0}^{n} q_{i, 0} \pi_{i} .
$$

Since

$$
\sum_{i=1}^{n} \sum_{j=1}^{n}\left(\lambda_{i}-\lambda_{k}\right) \pi_{i} \pi_{k}=0 \text {, by symmetry, }
$$

and since

$$
\left|\sum_{i=1}^{n}\left(\lambda_{i}-\lambda_{0}\right) \pi_{i} \pi_{0}\right|^{2}=\pi_{0}^{2}\left|\sum_{i=1}^{n}\left(\lambda_{i}-\lambda_{0}\right) \pi_{i}\right|^{2},
$$

we obtain upon recalling (1.1), that

$$
-q_{0}^{*}-K_{1} \pi_{0}^{2} \leqq M(\log r) \leqq-K_{2} \pi_{0}^{2}+\left|q_{0,0}\right| \pi_{0} / r \quad\left(q_{0}^{*}=\max _{1 \leqq i \leqq n} q_{i, 0}\right),
$$

where $K_{1}, K_{2}$ are positive constants depending only on the $\lambda_{i}$.

To prove the second part of (7.2), consider the function, in $\tilde{\Pi}=\Pi_{n+1} \cap\{r<1 /(n+1)\}$,

$$
v(r)= \begin{cases}a_{0} r & \text { if } 0 \leqq r \leqq R, \\ c \delta \log r+A-r & \text { if } \quad R<r \leqq \frac{1}{n+1}\end{cases}
$$

where $A, R, \delta$ are positive constants. We choose $A, R$ as of $\delta$ so that $v$ becomes $C^{1}$ at $r=R$, i.e.,

$$
\frac{c \delta}{R}-1=a_{0}, \quad c \delta \log R+A-R=a_{0} R ;
$$

$\delta$ is a positive constant (independent of $c$ ) to be determined. Thus 


$$
\begin{gathered}
R=\frac{c \delta}{a_{0}+1} \quad\left(R<\frac{1}{n+1} \text { if } c \text { is sufficiently small }\right), \\
A=c \delta+c \delta \log \frac{a_{0}+1}{c \delta} .
\end{gathered}
$$

Notice that $\widetilde{I} \subset G_{1}$. The condition

$$
v\left(\frac{1}{n+1}\right)<0
$$

is satisfied if

$$
c \delta \log \frac{1}{n+1}+A-\frac{1}{n+1}<0,
$$

i.e., (in view of (7.7), if $c$ is sufficiently small. We also easily find that

$$
v \leqq c \delta \log \left(a_{0}+1\right)
$$

Using (7.5), (7.7), (7.9) and the conditions (1.9), we find that, if $R<r<1 /(n+1)$,

$$
M v-\alpha v \geqq-K_{1} \frac{c \delta}{(n+1)^{2}}-\alpha c \delta \log \left(a_{0}+1\right)>-c
$$

provided $\hat{o}$ is sufficiently small (independently of $c$ ). We also have

$$
v<a_{0} r=g \quad \text { if } \quad R<r<\frac{1}{n+1} .
$$

Thus, we can apply Lemma 3.1 with $\tilde{u}=v$ and conclude that $v \leqq u$ in $\widetilde{I}$. Since $v<g$ if $r>R$, the same is true for $u$. Thus $S \cap G_{0}$ is contained in $N_{\delta c}$. Similarly one can prove that $S \cap G_{i}$ is contained in $N_{i c}$ for any $i \geqq 1$.

To prove the first part of (7.2), let

$$
w_{0}(r)=\left\{\begin{array}{lll}
a_{0} r & \text { if } & 0 \leqq r \leqq R_{0} \\
c \delta \log r+A_{0} & \text { if } & R_{0}<r \leqq 1
\end{array}\right.
$$

This function is $C^{1}$ at $r=R_{0}$ if

$$
\begin{aligned}
& R_{0}=\frac{c \delta}{a_{0}}, \\
& A_{0}=c \delta+c \delta \log \frac{a_{0}}{c \delta} .
\end{aligned}
$$

Using (7.5) and (1.9) we get, for $R_{0}<r<n /(n+1$,) 


$$
M w_{0}-\alpha w_{0} \leqq-\frac{K_{2} c \delta}{(n+1)^{2}}<-c
$$

if $\delta$ is sufficiently large (independently of $c$ ).

Similarly, we define functions $w_{i}$ for each $1 \leqq i \leqq n$ and take

$$
w=\min _{0 \leq i \leqq n} w_{i} .
$$

Note that if $r=n /(n+1)$ then certainly $w<w_{0}$. Thus, if $w=w_{0}$ then $r<n /(n+1)$ and, consequently,

$$
M w_{0}-\alpha w_{0}<-c \text { if further } r>R_{0} .
$$

The corresponding result is true for each $w_{2}$.

It follows that outside the $(c \delta)$-neighborhoods of the vertices $e_{i}$,

$$
M w-\alpha w<-c
$$

where $M w$ is taken in the distribution sense.

We can now apply Lemma 3.1 (whose proof extends, by approximation, to the case where $\tilde{u}$ is only Lipschitz continuous and $M \tilde{u}$ is taken in the distribution sense). It follows that $u \leqq w$, and the first part of (7.2) is established.

REMARK 1. The proof of the second part of (7.2) extends to the case where, for some $i$,

$$
\left|q_{i, i}\right| \geqq(n+1) \max _{k \neq i}\left(q_{k, 2}-\alpha\right) ;
$$

it gives the relation

$$
S \cap G_{i} \subset N_{\delta_{2} c}
$$

REMARK 2. From the proof of the first part of (7.2) we see that the function

$$
W_{0}(r)=\log \frac{r}{\varepsilon} \quad\left(r=\sum_{i=1}^{n} \pi_{i}, \varepsilon>0\right)
$$

satisfies $M W_{0} \leqq-A$ if $\varepsilon<r<n /(n+1)$, where $A$ is a positive constant independent of $\varepsilon$. Define $W_{i}$ in a similar manner with respect to the vertex $e_{i}$, and set

$$
W=\frac{1}{A} \min _{0 \leq i \leq n} W_{i} .
$$

Then $M W \leqq-1$ in

$$
\widetilde{N}_{\varepsilon}=\Pi_{n+1} \backslash N_{\varepsilon} .
$$


Also $W=0$ on $\partial \tilde{N}_{\varepsilon} \cap \stackrel{\circ}{\Pi}_{n+1}$. Denoting by $\tau_{\varepsilon}$ the hitting time of $N_{\varepsilon}$ by the process $\pi(t)$ it follows, by comparison, that

$$
E^{\pi} \tau_{\varepsilon} \leqq W(\pi)
$$

Thus

$$
E^{\pi} \tau_{\varepsilon} \leqq A_{1} \log \frac{1}{\varepsilon} \text { for all } \pi \in \Pi_{n+1}
$$

In the following section we shall obtain a more precise result as $\varepsilon \rightarrow 0$.

REMARK 3. From Theorem 7.1 it follows that

$$
u(\pi) \leqq A_{2} c
$$

for all $c$ sufficiently small, where $A_{2}$ is a constant independent of $c$. In the following section we shall obtain a more precise result as $c \rightarrow 0$.

8. Asymptotic estimates for $c \rightarrow 0$ (continued).

THEOREM 8.1. Suppose (1.9) holds. Then, for any $\pi \in \Pi_{n+1}$,

(8.1) $E^{\pi} \tau_{\varepsilon}=\left(\sum_{i=0}^{n} \gamma_{i} \pi_{i}\right) \log \frac{1}{\varepsilon}+0\left(\left(\log \frac{1}{\varepsilon}\right)^{1 / 2}\right), \frac{1}{2} \gamma_{i}=\left\{\min _{k \neq i}\left|\lambda_{k}-\lambda_{i}\right|\right\}^{-1}$

as $\varepsilon \rightarrow 0$.

The analogous result for discrete processes is given, for instance, in Kiefer and Sacks [10].

Proof. Set $\tau=\tau_{\varepsilon}$. Then $\tau$ is the first time $t$ such that

$$
\max _{0 \leqq j \leqq n} \pi_{j}(t)=1-\varepsilon .
$$

Using (1.11), the last inequality becomes

$$
\max _{0 \leqq j \leqq n}\left\{1+\sum_{k \neq j} \frac{\pi_{l k}}{\pi_{j}} e^{\left(\lambda_{k}-\lambda_{j}\right) \cdot \xi(t)-1 / 2\left(\left|\lambda_{k}\right|^{2}-\left|\lambda_{j}\right|^{2}\right)}\right\}^{-1}=1-\varepsilon,
$$

or

$$
\min _{0 \leq j \leq n} \max _{k \neq j} e^{\left(\lambda_{k}-\lambda_{j}\right) \cdot \xi(t)-1 / 2\left(\left|\lambda_{k}\right|^{2}-\left|\lambda_{j}\right|^{2}\right)}=C \varepsilon
$$

where $C$ is a random variable, $B_{1} \leqq C \leqq B_{2}$, and $B_{1}, B_{2}$ are positive constants independent of $\varepsilon$ (but depending on the initial point $\pi$. Taking the logarithm on both sides of (8.2) we conclude that 
(8.3) $\min _{0 \leqq j \leqq n} \max _{k \neq j}\left\{\left(\lambda_{k}-\lambda_{j}\right) \cdot \xi(\tau)-\frac{1}{2}\left(\left|\lambda_{k}\right|^{2}-\left|\lambda_{j}\right|^{2}\right) \tau\right\}=-\log \frac{1}{\varepsilon}+0(1)$.

Recalling that

$$
E^{\pi} \tau=\sum_{l=0}^{n} \pi_{l} E^{l} \tau,
$$

we proceed to evaluate $E^{l} \tau$ for a fixed $l$. With respect to the probability $P^{l}$,

$$
\xi(t)=w(t)+\lambda_{l} t \text { a.s. }
$$

Thus, the stopping time $\tau$ is the hitting time of some region $Q$ by the process $w(t)$.

We claim that for any hitting time $\tau$ of a region $Q$,

$$
E_{x}|w(\tau)|^{2}=2 n E_{x} \tau+|x|^{2}
$$

Indeed, if $Q^{c}=R^{n} \backslash Q$ is a bounded open set then, since both sides of (8.6) are harmonic functions in $Q^{c}$ taking the same boundary values $|x|^{2}$ on $\partial Q^{c}$, they must agree in $Q^{c}$. If $Q^{c}$ is unbounded then (8.6) follows by approximating $Q^{c}$ by bounded open sets.

From (8.6) applied with $x=0$ it follows that

$$
E^{l}|w(\tau)|=(2 n)^{1 / 2}\left(E_{x} \tau\right)^{1 / 2} \leqq C_{0}\left(\log \frac{1}{\varepsilon}\right)^{1 / 2}\left(C_{0} \text { constant }\right)
$$

where (7.11) was used.

Combining (8.3) with (8.5) and using (8.7), we find that

$$
\begin{gathered}
\min _{0 \leqq j \leqq n} \max _{k \neq j}\left\{\left(\lambda_{k}-\lambda_{j}\right) \cdot \lambda_{l}-\frac{1}{2}\left(\left|\lambda_{k}\right|^{2}-\left|\lambda_{j}\right|^{2}\right)\right\} E^{l} \tau \\
=-\log \frac{1}{\varepsilon}+0(1)+0\left(\left(\log \frac{1}{\varepsilon}\right)^{1 / 2}\right) .
\end{gathered}
$$

Next, one easily checks that

$$
\max _{k \neq j}\left\{\left(\lambda_{k}-\lambda_{j}\right) \cdot \lambda_{l}-\frac{1}{2}\left(\left|\lambda_{l}\right|^{2}-\mid \lambda_{l}\right)\right\}=\left\{\begin{array}{c}
\max _{k \neq l}\left[-\frac{1}{2}\left|\lambda_{k}-\lambda_{l}\right|^{2}\right] \text { if } j=l \\
\left(\lambda_{l}-\lambda_{j}\right) \cdot \lambda_{l}-\frac{1}{2}\left(\left|\lambda_{l}\right|^{2}-\left|\lambda_{j}\right|^{2}\right) \\
=\frac{1}{2}\left|\lambda_{l}-\lambda_{j}\right|^{2} \text { if } j \neq l .
\end{array}\right.
$$

Using this in (8.8), we obtain

$$
\left[\frac{1}{2} \min _{k \neq l}\left|\lambda_{k}-\lambda_{l}\right|^{2}\right] E^{l} \tau=\log \frac{1}{\varepsilon}+0\left(\left(\log \frac{1}{\varepsilon}\right)^{1 / 2}\right) ;
$$


recalling (8.4), the assertion (8.1) follows.

We wish to study the behavior of the solution $u(\pi)$ in a neighborhood of a vertex $e_{i}$ as $c \rightarrow 0$. It suffices to take $i=0$. It will be convenient to use the coordinates (3.3). We also set

$$
\begin{gathered}
u(y)=u(\pi), \quad|y|=y_{1}+\cdots+y_{n}, \\
L_{0} u \equiv \frac{1}{2} \sum_{i, j=1}^{n} \mu_{i j} y_{i} y_{j} \frac{\partial^{2} u}{\partial y_{i} \partial y_{j}} .
\end{gathered}
$$

The function $u(y)$ satisfies the variational inequality, in $0 \leqq|y|<\delta_{0}$, $\delta_{0}=1 /(n+1)$,

$$
\begin{gathered}
L_{0} u+\frac{1}{Y} \sum_{i, j=1}^{n} \mu_{i j} y_{i} y_{j} \frac{\partial u}{\partial y_{j}}-\alpha u+c \leqq 0, \\
u \leqq \frac{a_{0}|y|}{Y}, \\
\left(L_{0} u+\frac{1}{Y} \sum_{i, j=1}^{n} \mu_{i j} y_{i} y_{j} \frac{\partial u}{\partial y_{j}}-\alpha u+c\right)\left(u-\frac{a_{0}|y|}{Y}\right)=0 .
\end{gathered}
$$

Consider the variational inequality in $R_{n}^{+}$;

$$
\begin{gathered}
L_{0} \tilde{u}-\alpha \tilde{u}+1 \geqq 0, \\
\tilde{u} \leqq a_{0}|y|, \\
\left(L_{0} \tilde{u}-\alpha \tilde{u}+1\right)\left(\tilde{u}-a_{0}|y| \mid\right)=0,
\end{gathered}
$$

subject to the growth condition

$$
\widetilde{u}(y)=0(|y|) \text { as }|y| \longrightarrow \infty .
$$

TheOREM 8.2. Let (1.9) hold. Then there exists a unique solution $\widetilde{u}(y)$ of (8.11), (8.12); further, $0 \leqq u(y) \leqq C$ for some constant $C$, and

$$
\frac{u(c y)}{c} \longrightarrow \tilde{u}(y) \text { as } c \longrightarrow 0,
$$

uniformly in $y$ in compact subsets of int $R_{n}^{+}$.

Proof. For any $A>0$ let $\widetilde{u}_{A}$ be the solution of the variational inequality, in $|y|<A$,

$$
\begin{gathered}
L_{0} \tilde{u}_{A}-\alpha \tilde{u}_{A}+1 \geqq 0, \\
\tilde{u}_{A} \leqq a_{0}|y|, \\
\left(L_{0} \tilde{u}_{A}-\alpha \tilde{u}_{A}+1\right)\left(\tilde{u}_{A}-a_{0}|y|\right)=0, \\
\tilde{u}_{A}=0 \text { on }|y|=A .
\end{gathered}
$$

It is easily seen that 


$$
\widetilde{u}_{A} \geqq 0, \quad \widetilde{u}_{A}(y) \uparrow \quad \text { if } \quad A \uparrow
$$

It follows that

$$
\widetilde{u}(y) \equiv \lim _{A \uparrow \infty} \widetilde{u}_{A}(y)
$$

exists and it is a solution of the variational inequality (8.11).

We can represent $\tilde{u}_{A}(y)$ in the form

$$
\begin{gathered}
\tilde{u}_{A}(y)=\inf _{\tau} J_{y, A}(\tau), \\
J_{y, A}(\tau)=E_{y}\left[\int_{0}^{\tau \wedge \tau^{A}} e^{-\alpha t} d t+a_{0}|y(\tau)| e^{-\alpha \tau} I_{\tau<\tau^{A}}\right]
\end{gathered}
$$

where $y(t)$ satisfies

$$
d y(t)=\sigma(y(t)) d \widetilde{w}(t), \quad y(0)=y \quad\left(y \in \operatorname{int} R_{n}^{+}\right),
$$

for some $n$-dimensional Brownian motion $\widetilde{w}(t)$, and

$$
\sigma=\left(\sigma^{i j}\right), \quad \sigma^{i j}=\nu_{i j} y_{i}, \quad \sum_{k=1}^{n} \nu_{i k} \nu_{j k}=\mu_{i j}, \nu_{i j}=\nu_{j i} ;
$$

$\tau^{A}$ is the exit time of $y(t)$ from the set $|y| \leqq A$.

We claim that for all $1<A<\infty$,

$$
E_{y}\left[e^{-\alpha \tau^{A}}\right] \leqq \frac{C}{A^{\lambda}} \text { for some constants } \lambda>1, \quad C>0 .
$$

Indeed, by comparison

$$
E_{y}\left[e^{-\alpha \tau^{A}}\right] \leqq W(y)
$$

provided

$$
\begin{gathered}
L_{0} W-\alpha W \leqq 0 \text { for }|y|<A, \\
W \geqq 1 \text { on }|y|=A .
\end{gathered}
$$

Taking

$$
W(y)=\frac{C_{0}}{A^{\lambda}}\left(y_{1}^{\lambda}+\cdots+y_{n}^{\lambda}\right)
$$

where $C_{0}$ is a constant independent of $A$ and $\lambda>1, \lambda-1$ sufficiently small, the assertion (8.19) follows.

Taking $\tau=\tau^{A}$ in (8.17) and using (8.19) and (8.16), (8.15) we conclude that

$$
\widetilde{u}(y) \leqq C \quad(C \text { positive constant }) .
$$

Using (8.10) we find that the function 


$$
w_{c}(y)=\frac{u(c y)}{c}
$$

satisfies the variational inequality, in $0<y<\delta_{0} / c$,

$$
L_{0} w_{c}+\frac{c}{1+c|y|} \sum_{k, j=1}^{n} \mu_{i j} y_{i} y_{j} \frac{\partial w_{c}}{\partial y_{j}}-\alpha w_{c}+1 \geqq 0
$$

$$
w_{c} \leqq \frac{a_{0}|y|}{1+c|y|}
$$

$$
\left(L_{0} w_{c}+\frac{c}{1+c|y|} \sum_{k, j=1}^{n} \mu_{i j} y_{i} y_{j} \frac{\partial w_{c}}{\partial y_{j}}-\alpha w_{c}+1\right)\left(w_{c}-\frac{a_{0}|y|}{1+c|y|}\right)=0 \text {. }
$$

Hence we can write

$$
w_{c}(y)=\inf _{\tau} J_{y, A}^{c}(\tau)
$$

where

$$
\begin{aligned}
J_{y, A}^{c}(\tau)=E_{y}[ & \int_{0}^{\tau \wedge \bar{\tau}^{A}} e^{-\alpha t} d y+\frac{a_{0}\left|y_{c}(\tau)\right|}{1+c\left|y_{c}(\tau)\right|} e^{-\alpha \tau} I_{\tau<\bar{\tau} A} \\
& \left.+w_{c}\left(y_{c}\left(\bar{\tau}^{A}\right)\right) e^{-\alpha \bar{\tau} A} I_{\tau \geqq \bar{\tau}^{A}}\right]
\end{aligned}
$$

where $y_{c}(t)$ is the solution of the stochastic system

$$
d y_{c}(t)=\sigma\left(y_{c}(t)\right) d \tilde{w}(t)+b_{c}(t) d t, \quad y_{c}(0)=y,
$$

the matrix $\sigma(y)$ is defined above,

$$
b_{c}=\left(b_{c, i}\right), \quad b_{c, i}=\frac{c}{1+c|y|} \sum_{j=1}^{n} \mu_{i j} y_{i} y_{j},
$$

$\bar{\tau}^{A}=\tau^{A} \wedge \tau_{c}^{A}, \tau_{c}^{A}$ is the exit time of $y_{c}(t)$ from the set $|y| \leqq A$. Notice that $\bar{\tau}^{A}$ is a stopping time with respect to the $\sigma$-fields $\sigma(\widetilde{w}(s)$, $0 \leqq s \leqq t$ ), $t \geqq 0$; here $A$ is any fixed positive number $\leqq \delta_{0} / c$.

Analogously to (8.22), (8.23) we can write

$$
\tilde{u}(y)=\inf _{\tau} \widetilde{J}_{y, A}(\tau)
$$

where

$$
\begin{array}{r}
\widetilde{J}_{y, A}(\tau)=E_{y}\left[\int_{0}^{\tau \wedge \bar{\tau} A} e^{-\alpha t} d t+a_{0}|y(\tau)| e^{-\alpha \tau} I_{\tau<\bar{\tau} A}\right. \\
\left.+\widetilde{u}\left(y\left(\bar{\tau}^{A}\right)\right) e^{-\alpha \bar{\tau} A} I_{\tau \geqq \bar{\tau}^{A}}\right] .
\end{array}
$$

By standard arguments, for any large $T>0$ and small $\eta>0$,

$$
E_{y}\left[\sup _{0 \leqq t \leqq T}\left|y_{c}(t)-y(t)\right|^{2}\right] \leqq \eta^{2} \quad \text { if } \quad c \leqq c_{0}(\eta, T) .
$$


Next, the proof of (8.19) shows also that

$$
A^{\lambda} E_{y}\left[e^{-\alpha \tau_{c}^{A}}\right] \leqq C \text { for some } \lambda>1, C>0
$$

provided $c A \leqq 1 / C^{*}$ where $C^{*}$ is a suitably large positive constant (independent of $c, A$ ). Hence

$$
A^{\lambda} E_{y}\left[e^{-\alpha \bar{\tau} A}\right] \leqq C \text { provided } c A \leqq \frac{1}{C^{*}} .
$$

It follows that

$$
E_{y}\left[e^{-\bar{\alpha} \cdot A}\right]<\eta \quad\left(\text { if } \quad c \leqq \frac{1}{C^{*} A}\right)
$$

provided $A$ is sufficiently large.

Note that

$$
|y(\tau)| \leqq A, \quad\left|y_{c}(\tau)\right| \leqq A \text { if } \tau \leqq \bar{\tau}^{1} .
$$

Now fix $A$ such that (8.29) holds and then fix $T$ sufficiently large (depending on $A$ but not on $c$ ) such that

$$
\begin{gathered}
\left|y_{c}(\tau)\right|-\frac{\left|y_{c}(\tau)\right|}{1+c\left|y_{c}(\tau)\right|}<\eta \text { if } \tau \leqq \bar{\tau}^{A}, \\
A e^{-\alpha \tau}<\eta .
\end{gathered}
$$

Using (8.27), (8.29), (8.30) and (8.20), and recalling also (by (7.12)) that

$$
\left|w_{c}\right| \leqq A_{2},
$$

we deduce from (8.23), (8.26) that

$$
\left|J_{A, y}^{c}(\tau)-\widetilde{J}_{y, A}(\tau)\right| \leqq C \eta
$$

provided $c \leqq c_{*}(\eta, A) ; c_{*}$ and $C$ are independent of $\tau$ and $C$ is independent of $c, A$. Recalling (8.22), (8.25), we get

$$
\left|w_{c}(y)-\widetilde{u}(y)\right| \leqq C \eta,
$$

and the assertion (8.13) follows.

It remains to prove that any solution $\tilde{\tilde{u}}(y)$ of $(8.11),(8.12)$ must coincide with $\tilde{u}$. From (8.19) we conclude that

$$
E_{y}\left[\left|\tilde{\widetilde{u}}\left(y\left(\tau^{A}\right)\right)\right| e^{-\alpha \tau^{A}}\right] \longrightarrow 0 \text { if } A \longrightarrow \infty .
$$

Using (8.33) we can now repeat the argument which gave (8.32), with $w_{c}(y)$ replaced by $\widetilde{\widetilde{w}}(y)$. We thus deduce that 


$$
|\widetilde{\widetilde{u}}(y)-\tilde{u}(y)| \leqq C \eta \text { for and } \eta>0 ;
$$

hence $\tilde{\widetilde{u}}=\tilde{u}$.

From Remark 2 at the end of $\S 6$ we have that the component of the coincidence set of $w_{c}(y)$ which contains $y=0$ in convex. We also have:

THEOREM 8.3. The coincidence set $\widetilde{S}$ of $\tilde{u}(y)$ is a convex set.

By Caffarelli [5] it then follows that the free boundary $\partial \widetilde{S} \cap$ $\operatorname{int} R_{n}^{+}$is analytic.

Proof. It is easy to check that if $y \leftrightarrow \pi$ by (3.3) then $c y \leftrightarrow \pi^{c}=$ $\left(\pi_{0}^{c}, \cdots, \pi_{n}^{c}\right)$ where

$$
\pi_{0}^{c}=\frac{\pi_{0}}{\pi_{0}+c\left(1-\pi_{0}\right)}, \quad \pi_{i}^{c}=\frac{c \pi_{i}}{\pi_{0}+c\left(1-\pi_{0}\right)}(1 \leqq i \leqq n) .
$$

Setting $\widetilde{u}(\pi)=\widetilde{u}(y), u(\pi)=u(y)$ we then have, by Theorem 8.2,

$$
\frac{u\left(\pi^{c}\right)}{c} \longrightarrow \tilde{u}(\pi) \text { as } c \longrightarrow 0
$$

Set $\bar{\pi}=\pi-e_{0}, \bar{\pi}^{c}=\pi^{c}-e_{0}$. Then, as easily checked,

$$
\bar{\pi}^{c}=\frac{c}{1+(1-c) \bar{\pi}_{0}} \bar{\pi}
$$

and

$$
\bar{\pi}=\frac{1}{c+(c-1) \bar{\pi}_{0}^{c}} \bar{\pi}^{c}
$$

where

$$
\bar{\pi}=\left(\bar{\pi}_{0}, \cdots, \bar{\pi}_{n}\right), \quad \bar{\pi}^{c}=\left(\bar{\pi}_{0}^{c}, \cdots, \bar{\pi}_{n}^{c}\right) .
$$

Now, by the concavity of $u(\pi)$ established in Theorem 6.2, for any two points $\hat{\pi}$, $\hat{\pi}$ and $0<\lambda<1$,

$$
\frac{1}{c} \bar{u}\left(\lambda \hat{\bar{\pi}}^{c}+(1-\lambda) \hat{\overline{\hat{\pi}}}\right) \geqq \frac{1}{c}\left(\lambda \bar{u}\left(\hat{\bar{\pi}}^{c}\right)+\left(1-\lambda\left(\bar{u}\left(\hat{\bar{\pi}}^{c}\right)\right)\right.\right.
$$

where $\hat{\bar{\pi}}=\hat{\pi}-e_{0}$, $\hat{\bar{\pi}}=\hat{\hat{\pi}}-e_{0}$ and $\bar{u}(\bar{\pi})=u(\pi)$ for any $\pi$. We can write

$$
\lambda \hat{\bar{\pi}}^{c}+(1-\lambda) \hat{\overline{\bar{\pi}}}^{c}=\tilde{\tilde{\pi}}^{c}, \quad \tilde{\bar{\pi}}=\tilde{\pi}-e_{0},
$$

where, by (8.37), 


$$
\widetilde{\bar{\pi}}=\frac{\lambda \hat{\bar{\pi}}^{c}+(1-\lambda) \hat{\overline{\hat{\pi}}}^{c}}{c+(c-1)\left(\lambda \hat{\bar{\pi}}_{0}^{c}+(1-\lambda) \hat{\overline{\tilde{\pi}}}_{0}\right)} .
$$

The point $\tilde{\tilde{\pi}}$ depends on $c$; as $c \rightarrow 0$

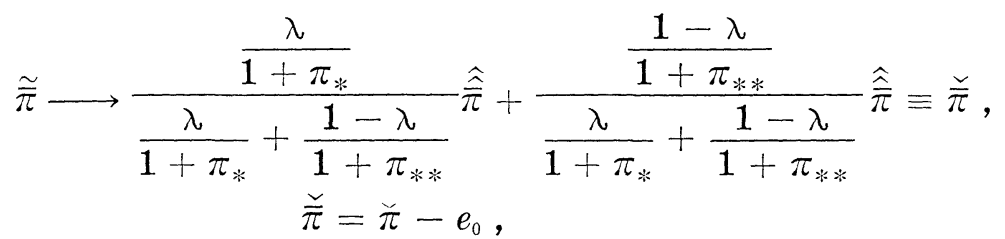

as seen using (8.36).

Using (8.35) we see that the right hand of (8.38) converges to

$$
\lambda \widetilde{u}(\hat{\pi})+(1-\lambda) \widetilde{u}(\hat{\tilde{\pi}}) \text { as } c \longrightarrow 0 .
$$

As for the left hand side, using (8.39)-(8.41) we find that it converges to $\overline{\widetilde{u}}(\check{\bar{\pi}})$, where $\overline{\widetilde{u}}(\bar{\pi})=\widetilde{u}(\pi)$ for any $\pi$. Hence

$$
\lambda \tilde{u}(\hat{\pi})+(1-\lambda) \tilde{u}(\hat{\tilde{\pi}}) \leqq \tilde{u}(\tilde{\pi})
$$

where $\check{\pi}$ is the same linear combination of $\pi$, $\hat{\bar{\pi}}$ as $\check{\pi}$ is of $\hat{\bar{\pi}}$, $\hat{\bar{\pi}}$ in (8.41). As $\lambda$ varies from 0 to 1 , the points $\check{\pi}$ fill the entire interval connecting $\hat{\pi}$ to $\hat{\pi}$.

For the obstacle of $\widetilde{u}$ we have equality in (8.42) (since it is a linear function). It follows that if $\hat{\pi}$ and $\hat{\hat{\pi}}$ are in the coincidence set of $\tilde{u}$, then so is the entire interval connecting them.

Since the coincidence set is convex in the $\pi$-space, it is also convex in the $y$-space.

We denote by $\widetilde{C}$ the continuation set for $\tilde{u}$.

Lemma 8.4. Suppose (1.9) holds and

$$
\mu_{1 j}=0 \text { for } 2 \leqq j \leqq n .
$$

Then

$$
\frac{\partial}{\partial y_{1}}\left(\widetilde{u}(y)-a_{0}|y|\right)<0 \text { in } \widetilde{C}
$$

Proof. Denote by $\hat{u}_{A}$ the solution of (8.14) subject to a different boundary condition, namely,

$$
\widehat{u}_{A}=0(A) \text {. }
$$

Representing $\hat{u}_{A}$ as a cost function and using (8.27), we find that

$$
\widehat{u}_{A}(y)-\widetilde{u}_{A}(y) \longrightarrow 0 \text { if } A \longrightarrow \infty \text {. }
$$


Hence

$$
\widehat{u}_{A}(y) \longrightarrow \widetilde{u}(y) \text { if } A \longrightarrow \infty
$$

Next, suppose we replace the domain $|y|<A$ by the domain

$$
\left\{y \in R_{n}^{+}, 0<y_{i}<A \text { for } 1 \leqq i \leqq n\right\}
$$

and denote by $u_{A}^{*}$ the solution of the variational inequality (8.14) in (8.46) subject to boundary condition (8.45). Then again we have

$$
u_{A}^{*}(y) \longrightarrow \widetilde{u}(y) \text { if } A \longrightarrow \infty \text {. }
$$

(This follows, for instance, by working throughout the proof of Theorem 8.2 with the domains (8.46) instead of the domains $|y|<A$.)

Denote by $u_{\tilde{\delta}, A}(y)(0<\delta<A)$ the solution of the variational inequality (8.14) in the domain

$$
\left\{y \in R_{n}^{+}, \delta<y_{i}<A \text { for } 1 \leqq i \leqq n\right\},
$$

subject to boundary conditions

$$
u_{\delta, A}(y)=0(A) .
$$

Then, for each fixed $A$, we clearly have

$$
u_{\hat{\delta}, A}(y) \longrightarrow u_{A}^{*}(y) \text { if } \delta \longrightarrow 0 .
$$

Set

$$
v=u_{\tilde{\delta}, A}-a_{0}|y| \text {. }
$$

We choose the boundary conditions in (8.49) such that $v \leqq 0$ and

$$
\begin{gathered}
v=0 \text { on } y_{1}=\delta \\
v_{y_{1}} \leqq 0 \text { on } y_{i}=\delta \text { and on } y_{i}=A \quad(2 \leqq i \leqq n) \\
v=-C^{*} A \text { on } y_{1}=A \quad\left(C^{*} \text { positive constant }\right)
\end{gathered}
$$

Consider the penalized problem corresponding to the variational inequality for $v$, namely,

$$
L_{0} v_{\varepsilon}-\alpha v_{\varepsilon}-\beta_{\varepsilon}\left(v_{\varepsilon}\right)+1-\alpha a_{0}|y|=0
$$

(where $\beta_{\varepsilon}$ is as in (2.3) and $\beta_{\varepsilon}(0)=1$ ). Using (8.43) and the condition $v_{\varepsilon}=0$ on $y_{1}=\delta$, we find that

$$
\mu_{11} \frac{\partial^{2} v_{\varepsilon}}{\partial y_{1}^{2}}=\beta_{\varepsilon}(0)+a_{0}|y|-1>0 \quad \text { if } \quad y_{1}=\delta
$$

and similarly 


$$
\mu_{11} \frac{\partial^{2} v_{\varepsilon}}{\partial y_{1}^{2}}=-\alpha C^{*} A+\beta_{\varepsilon}\left(-C^{*} A\right)+a_{0}|y|-1<0 \text { if } y_{1}=A
$$

provided $C^{*}$ is sufficiently large (independently of $A$ ).

Differentiating (8.53) with respect to $y_{1}$ and setting $z=\partial v_{\varepsilon} / \partial y_{1}$, we get

$$
L_{0} z-\alpha z-\beta_{\varepsilon}^{\prime}(v) z-\alpha a_{0}=0 .
$$

It follows that $z$ cannot take a positive maximum at an interior point. Furthermore, from (8.54), (8.55) we deduce that $z$ cannot take a maximum on the parts $y_{1}=\delta, y_{1}=A$ of the boundary. Since, by (8.52) $z \leqq 0$ in the remaining parts of the boundary, we conclude that

$$
z(y)<0 \text { in the domain (8.48) . }
$$

Taking $\varepsilon \rightarrow 0$ we get

$$
\frac{\partial}{\partial y_{1}}\left(u_{\delta, A}-a_{0}|y|\right)=\frac{\partial v}{\partial y_{1}} \leqq 0 .
$$

Taking $\delta \rightarrow 0$ and using (8.50), and then letting $A \rightarrow \infty$ and using (8.47), the assertion (8.44) follows.

THEOREM 8.5. Suppose (1.9) holds. Then the stopping set $\widetilde{\mathrm{S}}$ of $\tilde{u}$ contains an (int $R_{n}^{+}$)-neighborhood of the origin. If

$$
\mu_{i k}=0 \text { for some } i \text { and all } k \neq i
$$

then the free boundary $\widetilde{\Gamma}=\partial \widetilde{S} \cap\left(\right.$ int $R_{n}^{+}$) of $\tilde{u}$ can be represented in the form

$$
y_{i}=\varphi_{i}\left(y_{1}, \cdots, y_{i-1}, y_{i+1}, \cdots, y_{n}\right)
$$

where $\varphi_{i}$ is analytic.

The proof of the first part is the same as in the case of Theorem 3.2. To prove the second part, say for $k=1$, we use Lemma 8.4 and proceed as in $\S 5$.

REMark. Denote by $S_{c}$ the connected component of the coincidence set of $w_{c}(y)=u(c y) / c$ which contains $y=0$. Introduce the free boundaries

$$
\Gamma_{\mathrm{c}}=\partial S_{\mathrm{c}} \cap\left(\operatorname{int} R_{n}^{+}\right), \quad \widetilde{\Gamma}=\partial \widetilde{S} \cap\left(\operatorname{int} R_{n}^{+}\right)
$$

where $\widetilde{S}$ is the coincidence set for $\tilde{u}$. The sets $S_{c}, \widetilde{S}$ are contained in $|y|<R_{0}$ for some $R_{0}>0$. Introduce polar coordinates $(|y|, \theta)=$ 
$\left(|y|, \theta_{1}, \cdots, \theta_{n-1}\right)$ in $R_{n}^{+}$and a truncated convex cone

$$
K=\left\{y ; 0<|y| \leqq R_{0}, \theta \in G_{0}\right\} ;
$$

$G_{0}$ is such that $\partial K /\{0\}$ is contained in int $R_{n}^{+}$. Since $S_{c}$ and $\widetilde{S}$ are convex sets we can represent $\Gamma_{c}, \widetilde{\Gamma}$ in the form

$$
\Gamma_{c}:|y|=\rho_{c}(\theta) ; \widetilde{\Gamma}:|y|=\tilde{\rho}(\theta) .
$$

From Theorem 3.2 we deduce that, for any $\varepsilon>0$,

$$
\left|w_{c}(y)-\widetilde{u}(y)\right|<\varepsilon \quad \text { if } y \in K_{\delta}, \quad c \leqq c(\varepsilon, \delta),
$$

where $K_{\delta}$ is a $\delta$-neighborhood of $K$ intersected with int $R_{n}^{+} ; \delta>0$. We claim that

$$
\left|\rho_{c}(\theta)-\tilde{\rho}(\theta)\right|<C \varepsilon^{1 / 2} \quad \text { if } \quad \theta \in G_{0} ;
$$

this gives a rate of convergence of the free boundary of $u(c y) / c$ to that of $\widetilde{u}(y)$.

To prove $(8.60)$ note first that

$$
\begin{gathered}
K_{\delta} \cap \widetilde{S} \text { contains } S_{c, \varepsilon}=\left\{K_{\delta} \cap S_{c} \text { minus a } C \varepsilon^{1 / 2}\right. \text {-neighborhood of } \\
\left.K \cap S_{c}\right\} .
\end{gathered}
$$

Indeed, if $y \notin K_{\delta} \cap \widetilde{S}$ then (cf. [5])

$$
\sup _{B} \tilde{u}>\varepsilon
$$

where $B$ is a ball with center $y$ and radius $C \varepsilon^{1 / 2}$; hence, by (8.59), $\sup _{B} w_{c}>0$, i.e., $y \notin S_{c, \varepsilon}$.

Next $\rho_{c}(\theta)$ is uniformly Lipschitz in $\theta$ for $(|y|, \theta)$ in $K_{\delta / 2}$ and small $c$, since $K_{\delta} \cap S_{c}$ is convex and contains a fixed $K_{\delta}$-neighborhood of $y=0$. Also $\rho(\theta)$ is Lipschitz in $\theta$. These facts together with (8.61) and its counterpart with $\widetilde{S}, S_{c}$ interchanged, give the assertion (8.60) with a suitable $C$.

9. Asymptotic estimates when $c \rightarrow \infty$. Define, for any $\varepsilon>0$,

$$
D_{\varepsilon}=\left\{\varepsilon \text {-neighborhood of the ridge } \cap \cap \Pi_{n+1}\right. \text {. }
$$

THEOREM 9.1. Suppose that (1.9) holds and $c \geqq \alpha a_{i}(0 \leqq i \leqq n)$. Then there exist positive constants $B, c^{*}$ independent of $\alpha$, such that, if $c>c^{*}$,

$$
C \text { is contained in } D_{B / c} \text {. }
$$

Thus

(9.3) $u(\pi)=g(\pi)$ outside the B/c-neighborhood of the ridge . 
Proof. Suppose $\pi^{0}=\left(\pi_{0}^{0}, \pi_{1}^{0}, \cdots, \pi_{n}^{0}\right)$ is in $G_{0}$ and dist. $\left(\pi^{0}, R\right)=$ $B / c$. Let

$$
w(\pi)=-\delta c\left|\tilde{\pi}-\tilde{\pi}^{0}\right|^{2}+a_{0}\left(1-\pi_{0}\right)
$$

where $\tilde{\pi}=\left(\pi_{1}, \cdots, \pi_{n}\right), \tilde{\pi}^{0}=\left(\pi_{1}^{0}, \cdots, \pi_{n}^{0}\right)$. Clearly $w$ lies below the obstacle $g$ in $G_{0}$. Since

$$
\operatorname{dist.}\left(\pi^{0}, G_{i}\right)>\frac{B}{c} \quad(i \geqq 1),
$$

in each $G_{i} w$ decreases at a rate

$$
\geqq \delta c\left|\tilde{\pi}-\tilde{\pi}^{0}\right|-a_{0} \geqq B \delta-a_{0}>A_{0} \quad\left(A_{0}=\max _{0 \leqq i \leqq n} a_{i}\right)
$$

provided

$$
B \delta>2 A_{0} \text {. }
$$

This rate of decrease is faster than the linear rate of decrease of the obstacle $g$ in $G_{i}$. Hence $w$ lies below $g$.

Next, $w<0$ outside some $\left(A_{1} / \sqrt{c}\right)$-neighborhood $N$ (in $\left.\Pi_{n+1}\right)$ of $\pi^{0}$. We now compare $w$ with $u$ in $N$. By the calculation leading to $(7.5)$ we find that

$$
M w-\alpha w>-K_{1} c \delta \pi_{0}^{2}-\alpha w>-c
$$

if $\delta$ is sufficiently small independently of $c$; we use the fact that

$$
\alpha w \leqq \alpha a_{0}\left(1-\pi_{0}\right) \leqq \frac{3}{4} \alpha a_{0} c \leqq \frac{3}{4} c \text { in } N
$$

Since $u \geqq w$ on $\partial N$, Lemma 3.1 implies that $u \geqq w$ in $N$.

Since $w=g$ at $\pi^{0}$ it follows also that $u\left(\pi^{0}\right)=g\left(\pi^{0}\right)$ provided $\delta$ is sufficiently small and provided (9.4) holds. This completes the proof of (9.2) for points in $C \cap G_{0}$; the proof for $C \cap G_{i}(i \geqq 1)$ is similar.

Denote by $\widetilde{G}$ any compact subset of $\check{I}_{n+1}$ and set

$$
\widetilde{D}_{\varepsilon}=\{\varepsilon \text {-neighborhood of the ridge }\} \cap \breve{G} \text {. }
$$

THEOREM 9.2. There exists a positive constant $A$ (depending on G) such that, for all e sufficiently large,

$$
C \text { contains } \widetilde{D}_{A / c} \text {. }
$$

The proof is similar to the proof of a corresponding result in $[6 ; \S 4]$ for the elastic-plastic torsion problem.

Proof. Suppose $\pi^{0} \in \widetilde{D}_{A / c} \cap G_{0}$ and $\pi^{0} \notin C$. Suppose for simplicity 
that $\pi^{0}$ is close to $G_{1}$ at least as much as it is to any other $G_{i}$, $i \geqq 1$. Take points $\pi^{1} \in G_{0}, \pi^{2} \in G_{1}$ such that $\pi^{0}$ is the center of the segment $\overline{\pi^{1} \pi^{2}},\left|\pi^{1}-\pi^{2}\right|=A_{0} \sigma, \sigma=\operatorname{dist} .\left(\pi^{0}, R\right) ; A_{0}$ is chosen so that

$$
-\left[g\left(\pi^{2}\right)+g\left(\pi^{1}\right)-2 g\left(\pi^{0}\right)\right] \geqq A_{1} \sigma ;
$$

both $A_{0}, A_{1}$ are positive constants depending only on $a_{0}, a_{1}$. Since $u\left(\pi^{1}\right) \leqq g\left(\pi^{1}\right), u\left(\pi^{2}\right) \leqq g\left(\pi^{2}\right), u\left(\pi^{0}\right)=g\left(\pi^{0}\right)$, we obtain

$$
A_{1} \sigma \leqq-\left[u\left(\pi^{2}\right)+u\left(\pi^{1}\right)-2 u\left(\pi^{0}\right)\right] \leqq A_{2} \sigma^{2}|u|_{W^{2, \infty}(N)},
$$

where $N$ is some neighborhood of $\pi^{0}$. By standard estimates for variational inequalities [4], the right hand side is bounded by $A_{3} \sigma^{2} c$; here $A_{2}, A_{3}$ are positive constants independent of $c$. It follows that $\sigma \geqq 1 /\left(A_{3} c\right)$, and the proof is complete.

10. The case $\alpha=0$. For simplicity we shall assume in this section that (1.9) holds. Since $c>0$, if $E^{\pi} \tau$ is sufficiently large then $J_{\pi}(\tau)>V(\pi)$. Thus we may write

$$
V(\tau)=\inf _{E^{\pi_{\tau}<K_{0}}} J_{\pi}(\tau)
$$

where $K_{0}$ is some sufficiently large positive constant (depending on $c$ ).

The existence of a bounded solution (and, in fact, uniformly continuous in $\stackrel{\circ}{n+1}_{n+1}$ ) for the variational inequality (2.1) with $\alpha=0$ is proved in the same way as for $\alpha>0$. Theorem 3.2 remains valid with the same proof when $\alpha=0$. Defining $\tilde{\tau}$ by (2.12) and recalling (7.11) we clonclude that $E^{\pi} \widetilde{\tau}<\infty$. But then we can apply Ito's formula in order to deduce that $u(\pi)=J_{\pi}(\widetilde{\tau})$. We also get, by Ito's formula,

$$
u(\pi) \leqq J_{\pi}(\tau)
$$

for any stopping time $\tau$ with $E^{\pi} \tau<K_{0}$. Using (10.1) we deduce that

$$
u(\pi)=V(\pi)=J_{\pi}(\widetilde{\tau}) \quad \text { if } \quad \pi \in \stackrel{\circ}{n+1}_{n} .
$$

This proves the uniqueness of the solution $u$ of (2.1) when $\alpha=0$.

Using (10.1), the proof of Theorem 6.1 can be extended with minor changes to the case $\alpha=0$. Theorem 6.2 remains valid with the same proof.

The results of $\S \S 4,5,7,9$ extend without any changes to the case $\alpha=0$; instead of (7.12) we now have

$$
u(\pi) \leqq A_{2} c \log \frac{1}{c} .
$$


From Theorem 8.1 we deduce (for $\alpha=0$ ) that

$$
V(\pi)=\left(\sum_{i=0}^{n} \gamma_{i} \pi_{i}\right) c \log \frac{1}{c}+0\left(c\left(\log \frac{1}{c}\right)^{1 / 2}\right) \text { as } c \longrightarrow 0 .
$$

To generalize Theorem 8.2, consider the variational inequality (8.11) for $\alpha=0$ :

$$
\begin{gathered}
L_{0} \tilde{u}+1 \geqq 0, \\
\tilde{u} \leqq a_{0}|y|, \\
\left(L_{0} \tilde{u}+1\right)\left(\tilde{u}-a_{0}|y|\right)=0
\end{gathered}
$$

in $R_{n}^{+}$. A trivial solution is given by $a_{0}|y|$. We exclude this solution by requiring that

$$
\widetilde{u}(y)=O\left(\mid y^{\theta}\right) \text { for some } 0<\theta<1 .
$$

THEOREM 10.1. Let (1.9) hold. Then there exists a unique solution $\tilde{u}$ of (10.5), (10.6); further,

$$
0 \leqq \widetilde{u}(y) \leqq C \log (|y|+1)
$$

for some positive constant $C$, and

$$
\frac{u(c y)}{c} \longrightarrow \tilde{u}(y)
$$

uniformly in $y$ in compact subsets of int $R_{n}^{+}$.

Proof. Let

$$
z(y)=\left\{\begin{array}{lll}
a_{0}|y| & \text { if } & |y| \leqq \delta \\
A \log |y|+B & \text { if } & |y|>\delta
\end{array}\right.
$$

For suitable positive constants $\delta, A, B$, one finds that $z$ is a supersolution, i.e., $L_{0} z+1 \leqq 0$. Hence

$$
0 \leqq \tilde{u}_{A}(y) \leqq z(y)
$$

where $\widetilde{u}_{A}$ is the solution of (8.14) with $\alpha=0$. It follows that

$$
0 \leqq \tilde{u}(y) \leqq C \log (|y|+1), \quad \tilde{u}(y)=\lim \tilde{u}_{A}(y),
$$

where $C$ is a generic positive constant independent of $c$.

Next

$$
u(\pi) \leqq w_{0}(\boldsymbol{r}),
$$

where $w_{0}$ appears in (7.10). Recalling the precise form of $w_{0}(r)$ we compute that 


$$
w_{c}(y)=\frac{u\left(\pi^{c}\right)}{c} \leqq \frac{w_{0}\left(\pi^{c}\right)}{c} \leqq C \log (|y|+1)
$$

provided $|y| \leqq \delta_{0} / c$ where $\delta_{0}$ is any positive constant (independent of $c)$.

We are now ready to proceed with the proof of $(8.31),(8.32)$ in the case $\alpha=0$. From (10.9), (10.10) and the form of the cost functionals corresponding to $\widetilde{u}, w_{c}$ we see that we may restrict the $\tau$ to satisfy

$$
\tau \leqq \bar{\tau}^{A}, \quad E_{y} \tau \leqq C \log (|y|+1) \leqq C \log (A+1) .
$$

The last term in (8.23), for $\alpha=0$, is bounded by

$$
I_{A}=C \log (A+1) P_{y}\left[\tau^{A}<\tau\right] \text {. }
$$

Now, for any $\beta>0$,

$$
\begin{aligned}
P_{y}\left[\bar{\tau}^{A}<\tau\right] & =P_{y}\left[e^{-\beta(\bar{\tau} \cdot 1-\tau)}>1\right] \leqq E_{y}\left[e^{-\beta\left(\bar{\varepsilon}^{1}-\tau\right)}\right] \\
& \leqq\left\{E_{y}\left[e^{-\beta p \tau-1}\right]\right]^{1 / p}\left\{E_{y}\left[e^{\beta q \tau}\right]\right]^{1 / q}
\end{aligned}
$$

where $1 / p+1 / q=1, p>1, q>1$.

Since the stopping times which minimize the cost functions are exit times, we may take $\tau$ to be an exit time. Using the second inequality in (10.11) it then follows by $([8 ; \mathrm{p} .43])$ that

$$
E_{y}\left[e^{\beta q \tau}\right] \leqq C \quad \text { provided } \beta=\frac{1}{C \log (A+1)} .
$$

From the proof of (8.19) with $\lambda-1=p \beta / C$ we get

$$
E_{y}\left[e^{-\beta p \tau}\right] \leqq \frac{C|y|^{\lambda}}{A^{\lambda}} \leqq \frac{C|y|^{\lambda}}{A}
$$

substituting this estimate and (10.14) into (10.13), we get

$$
P_{y}\left[\bar{\tau}^{A}<\tau\right] \leqq \frac{C|y|^{2 / p}}{A^{1 / p}} .
$$

Consequently, from (10.12), for any $\eta>0$,

$$
I_{A}<\eta \quad \text { if } A \text { is sufficiently large ; }
$$

$A$ is independent of $c$. From now on $A$ is fixed. Hence, if $c$ is sufficiently large (depending on $A$ ),

$$
E_{y}\left|\frac{\left|y_{c}(\tau)\right|}{1+c\left|y_{c}(\tau)\right|}-\right| y_{c}(\tau)||<\eta \text {. }
$$

In order to complete the proof of (8.31), (8.32), it remains to show that 


$$
E_{y}\left\|y_{c}(\tau)|-| y(\tau)\right\|<\eta \text {. }
$$

Now, by (10.11), for any $T>0$,

$$
P_{y}[\tau>T] \leqq \frac{1}{T} E_{y} \tau \leqq \frac{C}{T} \log (|y|+1) .
$$

Hence, if $y$ varies in a compact subset,

$$
A E_{y}[\tau>T]<\eta \text { for a suitable } T>0 .
$$

Since, on the other hand, (8.27) holds, the estimates (10.16) follow if $c$ is small enough. We have thus completed the proof of (8.31), (8.32).

Suppose finally that $\widetilde{\widetilde{u}}$ is another solution of (10.5), (10.6). Repeating the preceding proof of (8.31), (8.32) with $w_{c}(y)$ replaced by $\widetilde{\widetilde{u}}(y)$ and choosing $p$ in (10.15) such that $1 / p>\theta$, we find that $\widetilde{\widetilde{u}} \equiv \tilde{u}$.

11. The case where $w(t)$ is $k$-dimensional. In this section we extend many of the results of the previous sections to the case where $w(t)$ is $k$-dimensional; the condition (1.1) is dropped. Thus the generator $M$ is generally a degenerate elliptic operator in the entire region $\Pi_{n+1}$. We assume, however, that (1.9) holds, so that

$$
J_{\pi}(\tau)=E^{\pi}\left[c \int_{0}^{\tau} e^{-\alpha t} d t+e^{-\alpha \tau} g(\pi(\tau))\right] .
$$

From (11.1), (1.15) and the strong Markov property we get

$$
\begin{gathered}
V(\pi)=\inf _{\tau \leqq \tau_{N}} E^{\pi}\left\{c \int_{0}^{\tau \wedge \tau_{N}} e^{-\alpha t} d t+e^{-\alpha \tau} g(\pi(\tau)) I_{\tau<\tau_{N}}\right. \\
\left.+e^{-\alpha \tau_{N}} V\left(\pi\left(\tau_{N}\right)\right) I_{\tau=\tau_{N}}\right\}
\end{gathered}
$$

for any stopping time $\tau_{N}$.

THEOREM 11.1. There exists a $\Pi_{n+1}^{0}$-neighborhood $\widetilde{S}_{i}$ of $e_{i}$ such that $\widetilde{S}_{i} \subset S$.

Proof. Set $W=V-g$ in a neighborhood $N$ of $e_{0}$ where $g(\pi)=$ $a_{0}\left(1-\pi_{0}\right)$, and let $\tau_{N}=$ exit time from $N$. Thus, for any stopping time $\tau \leqq \tau_{N}$

$$
\begin{aligned}
E^{\pi}\left[e^{-\alpha \tau} g(\pi(\tau))-g(\pi)\right] & =E^{\pi}\left[\int_{0}^{\tau} c e^{-\alpha t}(M-\alpha) g(\pi(t)) d t\right] \\
& =E^{\pi}\left[\int_{0}^{\tau} c e^{-\alpha t}(-\alpha g)(\pi(t)) d t\right] .
\end{aligned}
$$


Therefore, by (11.2),

$$
\begin{gathered}
W(\pi)=V(\pi)-g(\pi)=\inf _{\tau \leq \tau_{N}} E^{\pi}\left\{\int_{0}^{\tau \wedge \tau_{N}} e^{-\alpha t}(c-\alpha g)(\pi(t)) d t\right. \\
\left.+e^{-\alpha \tau_{N}} W\left(\pi\left(\tau_{N}\right)\right) I_{\tau=\tau_{N}}\right\} .
\end{gathered}
$$

Note also that $W \leqq 0$ and that $c-\alpha g \geqq C^{*}>0$ if $N$ is sufficiently small.

The function $z$ defined following (3.11) satisfies

$$
\begin{aligned}
M z-\alpha z+\gamma & \geqq 0 \quad(\text { with } \gamma<c-\alpha g), \\
z & \leqq 0, \\
(M z-\alpha z+\gamma) z & =0,
\end{aligned}
$$

and

$$
z \leqq w \text { on }|y|=R .
$$

Using Ito's formula we obtain

$$
z(\pi)=\inf _{\tau \equiv \tau_{N}} E^{\pi}\left[\int_{0}^{\tau \wedge \tau_{N}} e^{-\alpha t} \gamma d t+e^{-\alpha \tau_{N}} z\left(\pi\left(\tau_{N}\right)\right) I_{\tau=\tau_{N}}\right] .
$$

Comparing with (11.3) we conclude that

$$
z(\pi) \leqq W(\pi) .
$$

Since $z(\pi)=0$ where $\pi$ varies in some neighborhood of $e_{0}$, the same follows for $W$; this completes the proof.

Theorems 6.1, 6.2 remain valid with the same proof.

LEMMA 11.2. The estimate (7.11) is valid.

Proof. Because of the degeneracy of $M$, we need to choose the functions $W_{i}$ differently than in the proof of (7.11) in $\S 7$. For simplicity we exhibit the construction in case $n=2$. Take

$$
W_{0}=\log \frac{\pi_{1}+\pi_{2}}{\varepsilon}+\log \frac{\delta_{1} \pi_{1}+\delta_{2} \pi_{2}}{\varepsilon}
$$

outside an $\varepsilon$-neighborhood of $e_{0}$, where $\delta_{1}=1, \delta_{2}=3$. Thus $W_{0} \geqq 0$ and

$$
\begin{aligned}
M W_{0} & =M \log \left(\pi_{1}+\pi_{2}\right)+M \log \left(\delta_{1} \pi_{1}+\delta_{2} \pi_{2}\right) \\
& \leqq-\frac{A_{0}}{r^{2}}\left[\left(\pi_{1}-\pi_{2}\right)^{2}+\left(\delta_{1} \pi_{1}-\delta_{2} \pi_{2}\right)^{2}\right] \\
& \leqq-\frac{A\left(\pi_{1}+\pi^{2}\right)^{2}}{r^{2}}=-A
\end{aligned}
$$


where $r=\pi_{1}+\pi_{2}$ and $A_{0}, A$ are positive constants.

Similarly, we define the $W_{i}$ with respect to $e_{i}$ and notice that

$$
M W_{i} \leqq 0, \quad W_{i} \geqq 0 .
$$

Hence $W=\sum_{i=0}^{2} W_{i}$ satisfies $M W<-A, W>0$ outside an $\varepsilon$-neighborhood of the vertices. This implies, by Ito's,formula,

$$
E^{\pi} \tau_{\varepsilon} \leqq \frac{W}{A},
$$

and (7.11) follows.

Using (7.11) we can now derive Theorem 8.1 as before.

Theorem 8.2 asserts that

$$
\frac{u(c g)}{c} \longrightarrow \tilde{u}(g) \text { as } c \longrightarrow 0
$$

where $\tilde{u}(g)$ is defined by (8.25), (8.26). The proof can actually be given by comparing the cost functionals and without introducing variational inequalities at all. Notice that the crucial estimate (8.12) remains valid here (with the same proof) and that also the inequality

$$
\frac{u(c g)}{c} \leqq A
$$

which is needed in proving (11.4) is true (in fact, taking $\tau \rightarrow \infty$ in the cost functional which defines $u$ we obtain (11.5)).

The proof of Theorem 3.2 extends to $\widetilde{u}$ (cf. the proof of Theorem 11.1), showing that the stopping set $\widetilde{S}$ contains a neighborhood of each vertex. This proves the part

$$
S \supset N_{\delta_{1} c}
$$

of Theorem 7.1; the other part follows as in the proof of Theorem 7.1, since

$$
M(\log r) \geqq-K_{1} \pi_{0}^{2} .
$$

The convexity of $\widetilde{S}$ (Theorem 8.3) remains unchanged. Finally, the results of $\S 10$ (the case $\alpha=0$ ) extend with minor changes.

We shall now obtain additional information, taking $n=2$ and $w(t)$ to be 1-dimensional. We also take for simplicity

$$
\lambda_{0}=0, \quad \lambda_{1}=-1, \quad \lambda_{2}=1 .
$$

Writing $\pi_{i}(t)$ in terms of the observed process (see (1.11)) we easily compute that 


$$
\begin{gathered}
\xi(t)=\frac{1}{2} \log \frac{\pi_{1}}{\pi_{2}} \frac{p_{1}}{p_{2}} \\
t=\log \frac{\pi_{0}^{2}}{\pi_{1} \pi_{2}} \frac{p_{1} p_{2}}{p_{0}^{2}}
\end{gathered}
$$

where $p_{j}=\pi_{j}(0), \pi_{j}=\pi_{j}(t)$.

The mapping

$$
\sigma:(\xi(t), t) \longrightarrow \pi(t)
$$

is $1-1$, mapping the half-plane $t>0$ onto a subset of $\Pi_{3}$ defined by

$$
\pi_{0}^{2}>\pi_{1} \pi_{2} \frac{p_{0}^{2}}{p_{1} p_{2}}
$$

The ridge of $\Pi_{3}$ is not in the stopping set $S$ and $\sigma$ maps $S_{i}$ onto a set $\sum_{i}$; see the accompanying figure.
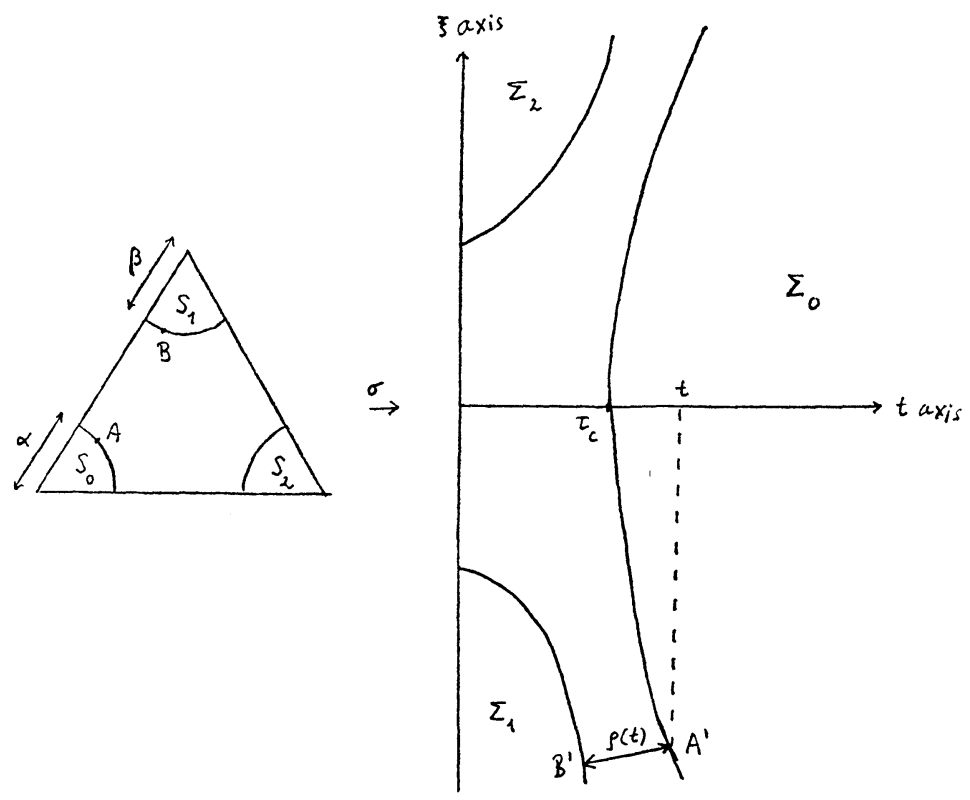

Take a point $t$ on the $t$-axis and mark the point $A^{\prime}=\left(\xi^{\prime}, t\right)$ on $\partial \Sigma_{0}$ with $\xi^{\prime}<0$. Denote by $\rho(t)$ the distance from $A^{\prime}$ to $\partial \Sigma_{1}$; it is achieved at $B^{\prime} \in \partial \Sigma_{1}$. Denote by $A, B$ the inverse images of $A^{\prime}, B^{\prime}$ under $\sigma$.

THEOREM 11.3. As $t \rightarrow \infty$

$$
\rho(t) \longrightarrow \frac{2}{\sqrt{5}}\left|\log \frac{\alpha(1-\beta)}{\beta(1-\alpha)}\right|
$$


where $\alpha, \beta$ are positive constants described in the previous figure.

Proof. Write

$$
A=\left(\pi_{0}, \pi_{1}, \pi_{2}\right), \quad B=\left(\bar{\pi}_{0}, \bar{\pi}_{1}, \bar{\pi}_{2}\right) .
$$

Then, as $t \rightarrow \infty$,

$$
\begin{aligned}
& \pi_{2} \longrightarrow 0, \quad \bar{\pi}_{2} \longrightarrow 0, \pi_{0} \longrightarrow \alpha, \\
& \pi_{1} \longrightarrow 1-\alpha, \quad \bar{\pi}_{0} \longrightarrow \beta, \quad \bar{\pi}_{1} \longrightarrow 1-\beta .
\end{aligned}
$$

Setting

$$
\pi_{2}=\varepsilon, \quad \pi_{2}=\tilde{\varepsilon},
$$

we can then write

$$
\begin{array}{ll}
\pi_{0}=\alpha-\gamma_{\varepsilon}, & \pi_{1}=1-\alpha+\gamma_{\varepsilon}-\varepsilon, \\
\bar{\pi}_{0}=\beta-\delta_{\bar{\varepsilon} \bar{\varepsilon}}, & \bar{\pi}_{1}=1-\beta+\delta_{\bar{\varepsilon} \bar{\varepsilon}}-\tilde{\varepsilon},
\end{array}
$$

where

$$
\varepsilon \longrightarrow 0, \tilde{\varepsilon} \longrightarrow 0, \gamma_{\varepsilon} \longrightarrow 0, \delta_{\varepsilon \tilde{\varepsilon}} \longrightarrow 0 \text { as } t \longrightarrow \infty .
$$

From (11.6) we find that

$$
\rho=\rho(t)=\left\{\frac{1}{4}\left[\log \frac{\pi_{2}}{\pi_{1}} \frac{\bar{\pi}_{1}}{\bar{\pi}_{2}}\right]^{2}+\left[\log \frac{\pi_{0}^{2}}{\pi_{1} \pi_{2}} \frac{\bar{\pi}_{1} \bar{\pi}_{2}}{\bar{\pi}_{0}^{2}}\right]^{2}\right\}^{1 / 2} .
$$

Hence

$$
\left.\rho^{2}=\frac{1}{4}\left[\log \left(\lambda \frac{1-\beta}{1-\alpha}(1+o(1))\right)\right]^{2}+\left[\log \frac{1}{\lambda} \frac{\alpha^{2}(1-\beta)}{\beta^{2}(1-\alpha)}(1+o(1))\right)\right]^{2}
$$

where $\lambda=\tilde{\varepsilon} / \varepsilon, o(1) \rightarrow 0$ as $t \rightarrow \infty$.

From the definition of $B^{\prime}$ it follows that

$$
\rho=\tilde{\rho}(1+o(1))
$$

where

$$
\tilde{\rho}=\min _{\lambda}\left\{\frac{1}{4}\left[\log \lambda \frac{1-\beta}{1-\alpha}\right]^{2}+\left[\log \lambda \frac{\beta^{2}(1-\alpha)}{\alpha^{2}(1-\beta)}\right]^{2}\right\}^{1 / 2} .
$$

Set

$$
\rho(\lambda)=\frac{1}{4}(\log k \lambda)^{2}+(\log h \lambda)^{2} \quad(k>0, h>0) .
$$

Then $\min \rho(\lambda)$ is obtained at

$$
\lambda=h^{-4 / 5} k^{-15} .
$$


Using this value in our special case of $\widetilde{p}$, (11.7) follows.

Consider next the point $(\xi, t)=\left(0, \tau_{c}\right)$ on $\partial \Sigma_{0}$.

THEOREM 11.4. As $c \rightarrow 0$

$$
\tau_{c} \sim \log \frac{1}{\gamma^{2} c^{2}}
$$

where $\gamma$ is a positive constant.

Proof. $\left(0, \tau_{c}\right)$ corresponds to $\left(\pi_{0}, \pi_{1}, \pi_{2}\right)$ where, by Theorem 8.2 and 8.3,

$$
\begin{aligned}
& \pi_{0} \sim \frac{1}{1+\gamma_{1} c+\gamma_{2} c} \\
& \pi_{i} \sim \frac{\gamma_{i} c}{1+\gamma_{1} c+\gamma_{2} c} \quad(i=1,2)
\end{aligned}
$$

and $\gamma_{1}=\gamma_{2}=\gamma$ since $\pi_{1}=\pi_{2}$. Since

$$
\tau_{c}=\log \frac{\pi_{0}^{2}}{\pi_{1} \pi_{2}}=\log \frac{\pi_{0}^{2}}{\pi_{1}^{2}}
$$

the assertion follows.

REMARK. Theorems 11.3, 11.4 have the advantage of providing direct information on the observed process $(\xi(t), t)$. In case $n>\mathbf{2}$ we get a similar picture with $n+1$ regions $\Sigma_{i}$ in the half-plane $t>0$ (of the $(\xi, t)$ variable).

\section{REFERENCES}

1. H. W. Alt, The fluid flow through porous media. Regularity of the free surface, Manuscripta Math., 21 (1977), 255-272.

2. R. F. Anderson and A. Friedman, Multi-dimensional quality control problems and quasi-variational inequalities, Trans. Amer. Math. Soc., 246 (1978), 31-76.

3. A. Bensoussan and J. P. Lions, Variationnelles en Controle Stochastique, Dunod, Paris, 1978.

4. H. Brezis and D. Kinderlehrer, The smoothness of solutions to nonlinear variational inequalities, Indiana Univ. Math. J., 23 (1974), 831-844.

5. L. A. Caffarelli, The regularity of free boundaries in higher dimensions, Acta Math., 139 (1977), 155-184.

6. L. A. Caffarelli and A. Friedman, The free boundary for elastic-plastic torsion problems, Trans. Amer. Math. Soc., 252 (1979), 65-97.

7. Y. S. Chow and H. Robbins, On optimal stopping rules, Z. Wahrscheinlichkeitstheorie und verw. Gebiete, 2, (1963), 33-49.

8. A. Friedman, Stochastic Differential Equations and Applications, vol. 1, Academic Press, New York, 1976.

9. —, Stochastic Differential Equations and Applications, vol. 2, Academic Press, New York, 1976. 
10. A. Friedman, On the free boundary of a quasi variational inequality arising in a problem of quality control. Trans. Amer. Math. Soc., 246 (1978), 95-110.

11. J. Kiefer and J. Sacks, Asymptotically optimum sequential inference and design, Ann. Math. Statistics, 34 (1963), 705-750.

12. D. Kinderlehrer and L. Nirenberg, Regularity in free boundary problems, Ann. Scu. Norm. Sup. Pisa, 4 (1977), 373-391.

13. E. Sh. Liptser and A. N. Shiryayev, Statistics of Random Processes, Springer Verlag, New York-Heidelberg-Berlin, 1977.

14. A. N. Shiryayev, Optimal Stopping Rules, Springer-Verlag, New York-HeidelbergBerlin, 1978.

15. A. Wald, Sequential Analysis, Wiley and Sons, New York, 1947.

Received July 12, 1979 and in revised form November 2, 1979. This work is partially supported by National Science Foundation Grants 7406375 A01 and MCS-881 7204.

UNIVERSITY OF MINNESOTA

MINNEAPOLIS, MN

AND

NORTHWESTERN UNIVERSITY

Evanston, IL 


\section{PACIFIC JOURNAL OF MATHEMATICS}

\section{EDITORS}

DONALD BABBITT (Managing Editor)

University of Galifornia

Los Angeles, California 90024

HUgo RossI

University of Utah

Salt Lake City, UT 84112

C. C. MOORE AND ANDREW OGG

University of California

Berkeley, CA 94720

\section{J. DUGUNDJI}

Department of Mathematics University of Southern California Los Angeles, California 90007

R. Finn and J. Milgram

Stanford University

Stanford, California 94305

\section{ASSOCIATE EDITORS}
R. ARENS
E. F. BECKENBACH
B. H. NeUManN
F. WOLF
K. YosHIDA

\section{SUPPORTING INSTITUTIONS}

UNIVERSITY OF ARIZONA

UNIVERSITY OF BRITISH COLUMBIA

CALIFORNIA INSTITUTE OF TECHNOLOGY

UNIVERSITY OF CALIFORNIA

MONTANA STATE UNIVERSITY

UNIVERSITY OF NEVADA, RENO

NEW MEXICO STATE UNIVERSITY

OREGON STATE UNIVERSITY
UNIVERSITY OF OREGON UNIVERSITY OF SOUTHERN CALIFONIA STANFORD UNIVERSITY UNIVERSITY OF HAWAII UNIVERSITY OF TOKYO UNIVERSITY OF UTAH WASHINGTON STATE UNIVERSITY UNIVERSITY OF WASHINGTON 


\section{Pacific Journal of Mathematics}

\section{Vol. 93, No. $1 \quad$ March, 1981}

Richard Arens, Reducing the order of a Lagrangian $\ldots \ldots \ldots \ldots \ldots \ldots \ldots$

Richard Arens, Manifestly dynamic forms in the Cartan-Hamilton treatment

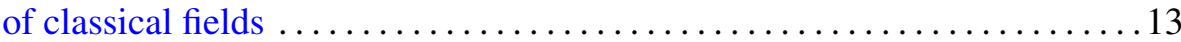

Jimmy T. Arnold, Power series rings over discrete valuation rings $\ldots \ldots \ldots 31$

Charles A. Asmuth and Joe Repka, Supercuspidal components of the

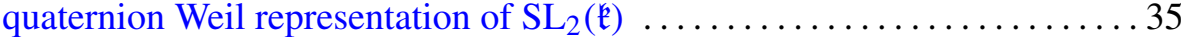

Luis A. Caffarelli and Avner Friedman, Sequential testing of several simple hypotheses for a diffusion process and the corresponding free boundary problem ................................. 49

William B. Jacob, Fans, real valuations, and hereditarily-Pythagorean fields .............................................. 95

W. J. Kim, Asymptotic properties of nonoscillatory solutions of higher order

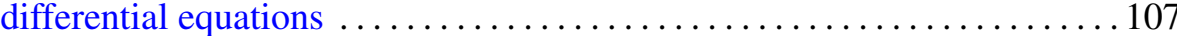

Wayne Steven Lewis, Embeddings of the pseudo-arc in $E^{2} \ldots \ldots \ldots \ldots \ldots 115$

Daniel Alan Marcus, Closed factors of normal Z-semimodules . ......... 121

Mitsuru Nakai and Leo Sario, Harmonic functionals on open Riemann surfaces ............................................. 147

John Currie Quigg, Jr., On the irreducibility of an induced

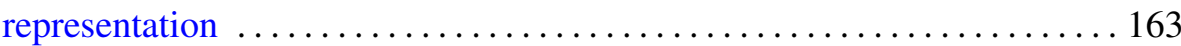

John Henry Reinoehl, Lie algebras and Hopf algebras 181

Joe Repka, Base change for tempered irreducible representations of $\mathrm{GL}(n, \mathbf{R})$

Peter John Rowley, Solubility of finite groups admitting a fixed-point-free automorphism of order $r s t$. I . . . . . . . . . . . . . . . . . . 201

Alan C. Woods, The asymmetric product of three homogeneous linear forms 